Copyright

by

Kelley Elizabeth Hutchins

2011 
The Thesis committee for Kelley Elizabeth Hutchins Certifies that this is the approved version of the following thesis:

\section{Detection and Transient Dynamics Modeling of Experimental Hypersonic Inlet Unstart}

APPROVED BY

SUPERVISING COMMITTEE:

Maruthi Akella, Supervisor

Noel Clemens 


\title{
Detection and Transient Dynamics Modeling of Experimental Hypersonic Inlet Unstart
}

\author{
by
}

Kelley Elizabeth Hutchins, B.S.

\author{
THESIS \\ Presented to the Faculty of the Graduate School of \\ The University of Texas at Austin \\ in Partial Fulfillment \\ of the Requirements \\ for the Degree of \\ MASTER OF SCIENCE IN ENGINEERING
}

THE UNIVERSITY OF TEXAS AT AUSTIN

December 2011 
To Milad, my family, and of course Vega 


\section{Acknowledgments}

I would like to thank my advisor, Dr. Maruthi Akella, for the opportunity to be part of his research group and contribute to the larger project that is the basis for this thesis. I also want to thank Dr. Akella's previous student Dr. Sukumar Srikant whose work and experience I relied on heavily when beginning my research as well as my current fellow group members who always keep the office exciting.

I would also like to thank Dr. Noel Clemens with the Center for Aeromechanics Research and each of his present and former students who have worked on the unstart problem. A significant portion of this thesis is based on either data that they have collected from hardware they maintain or on previous work they have conducted.

Finally, I would especially like to thank Dr. Jeffrey Donbar and others with the AFRL Propulsion Directorate at Wright-Patterson AFB who have provided me extensive advice, fielded numerous questions and requests, and permitted me access to their hypersonic test facilities and associated data. Their support of my summer internship at AFRL allowed me to quickly immerse myself in the unfamiliar topic of hypersonic propulsion and laid the groundwork for the work presented here. 


\title{
Detection and Transient Dynamics Modeling of Experimental Hypersonic Inlet Unstart
}

\author{
Kelley Elizabeth Hutchins, M.S.E. \\ The University of Texas at Austin, 2011
}

Supervisor: Maruthi Akella

\begin{abstract}
During unstart, the rapid upstream propagation of a hypersonic engine's inlet shock system can be clearly seen through inlet pressure measurements. Specifically, the magnitude of the pressure readings suddenly and dramatically increases as soon as the leading edge of the shock system passes the measurement location. A change detection algorithm can monitor the pressure time history at a given sensing location and determine when an abrupt pressure rise occurs. If this kind of information can be obtained at various sensing locations distributed throughout the inlet then a feedback control scheme has an improved basis upon which to make actuation decisions for preventing unstart. In this thesis a variety of change detection algorithms have been implemented and tested on multiple sources of experimental high-speed pressure transducer data. The performance of these algorithms is compared and suitability of each algorithm for the general unstart problem is discussed. Attempts to model the transient dynamics governing the unstart process have also been made through
\end{abstract}


the use of system identification techniques. The result of these system identification efforts is a partially nonlinear mathematical model that describes shock motion through pressure signals. The process reveals that the nonlinear behavior can be separated from the linear with relative ease. Related attempts are then made to create a model where the nonlinear portion has been specified leaving only the linear portion to be determined by system identification. The modeling and identification process specific to the unstart data used is discussed and successful models are presented for both cases. 


\section{Table of Contents}

Acknowledgments $\quad$ v

Abstract vi vi

List of Tables $\quad$ x

List of Figures $\quad$ xi

Chapter 1. Introduction 1

1.1 Overview ....................... 1

1.2 Related Work ................... 3

1.3 Contributions of Thesis . . . . . . . . . . . . . 5

Chapter 2. Change Detection 6

2.1 Data Sets . . . . . . . . . . . . . . . 7

2.2 CUSUM Algorithm . . . . . . . . . . . . . . . . 11

2.3 Other Algorithms . . . . . . . . . . . . . . . . . . 17

2.3.1 Power Spectrum Based Detection . . . . . . . . . . 17

2.3.2 Standard Deviation Based Detection . . . . . . . . . . 20

2.3.3 Pressure Magnitude Based Detection . . . . . . . . . . 22

2.4 Algorithm Performance . . . . . . . . . . . . . . 23

2.4.1 Filtering Comparison . . . . . . . . . . . 24

2.4.2 CUSUM Application across Data Sets . . . . . . . . 25

2.4.3 Multiple Algorithm Comparison . . . . . . . . . . 32

Chapter 3. Modeling Transient Dynamics with System Identification 35

3.1 Data Collection . . . . . . . . . . . . . . . 36

3.1.1 UT Hardware . . . . . . . . . . . . . . . . . 36 
3.1 .2 AFRL Hardware . . . . . . . . . . . . . . . . . . 38

3.2 System Identification . . . . . . . . . . . . . . . . . 44

3.2 .1 Overview . . . . . . . . . . . . . . . . . . . 44

3.2 .2 Model Selection . . . . . . . . . . . . . . . . 50

3.2 .3 Hammerstein-Wiener Model . . . . . . . . . . . . . . . 54

3.3 Results . . . . . . . . . . . . . . . . . 56

3.3 .1 UT Model . . . . . . . . . . . . . . . . . 56

3.3 .2 AFRL Model . . . . . . . . . . . . . . . . . . 59

Chapter 4. Modeling Transient Dynamics with Structured Nonlinearities $\quad 63$

4.1 Motivation . . . . . . . . . . . . . . . . . . . 63

4.2 Structure Simplification . . . . . . . . . . . . . . . 63

4.2 .1 Identify Nonlinear Effects . . . . . . . . . . . . . . . 64

4.2 .2 Simplified Model Structure . . . . . . . . . . . . 66

4.3 Application to AFRL Data . . . . . . . . . . . . . . . 69

4.3.1 Obtaining Linear and Nonlinear Elements . . . . . . 69

4.3 .2 Results . . . . . . . . . . . . . . . . 73

$\begin{array}{lll}\text { Chapter 5. Conclusions and Suggestions for Future Work } & 77\end{array}$

$\begin{array}{ll}\text { Appendices } & 79\end{array}$

Appendix A. UT Full System Identification Model 80

Appendix B. AFRL Full System Identification Model 82

$\begin{array}{lll}\text { Appendix C. } & \text { AFRL Simplified Model } & 86\end{array}$

$\begin{array}{ll}\text { Bibliography } & 89\end{array}$ 


\section{List of Tables}

2.1 Comparison of detection times for unfiltered data and low-pass filtered data $(100 \mathrm{~Hz}$ cutoff $)$. . . . . . . . . . . 25

2.2 Comparison of detection times for all algorithms . . . . . . . . 32

2.3 Summary of algorithm advantages and disadvantages . . . . . 34

A.1 10 unit piecewise nonlinearity for input 1 . . . . . . . . . 81

A.2 10 unit piecewise nonlinearity for input $2 \ldots \ldots$. . . . . . . 81

A.3 10 unit piecewise nonlinearity for output . . . . . . . . . . . 81

B.1 Sigmoid network parameter values for input 1 . . . . . . . 84

B.2 Sigmoid network parameter values for input 2 . . . . . . . 85 


\section{List of Figures}

2.1 Hardware schematic for Data Set 1 . . . . . . . . . . . . . 9

2.2 Hardware schematic for Data Set 2 . . . . . . . . . . . . . . 10

2.3 Hardware schematic for Data Set 3 . . . . . . . . . . . . . . 11

2.4 Hardware schematic for Data Set 4 . . . . . . . . . . . . . . . 12

2.5 Flowchart of CUSUM algorithm . . . . . . . . . . . . 15

2.6 Top: Original pressure signal Middle: Processed pressure signal Bottom: CUSUM metric and threshold . . . . . . . . . . . 16

2.7 Top: Power spectrum of pre-change signal Bottom: Power spectrum of signal during and after shock's pass exhibiting peak . 18

2.8 Top: Power spectrum of pre-change signal Bottom: Power spectrum of signal during and after shock's pass lacking peak . . . 19

2.9 Top: Original pressure signal Bottom: Normalized spectral power and threshold . . . . . . . . . . . . 21

2.10 Standard deviation of pressure signal with threshold . . . . . . 22

2.11 Pressure signal with threshold . . . . . . . . . . . . . . 23

2.12 CUSUM performance on Data Set 2 example, transducer K2,

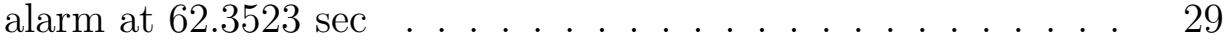

2.13 CUSUM performance on Data Set 3 example, transducer T2,

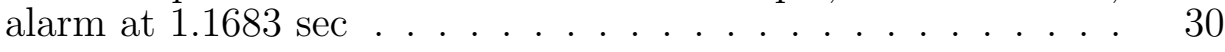

2.14 CUSUM performance on Data Set 4 example, transducer T5,

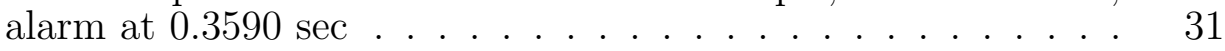

3.1 Pulse train created with flap movement . . . . . . . . . . . . . 37

3.2 Pulse train created with VGJ cycles . . . . . . . . . . . . . . . 38

3.3 Example of sinusoidal fueling pattern, input: equivalence ratio, output: pressure at selected transducer . . . . . . . . . 40

3.4 Sinusoid fit to sampled input data . . . . . . . . . . . . . 41

3.5 Step input created with direct valve manipulation . . . . . . . 42

3.6 Pulses created with air throttle manipulation . . . . . . . . . 43

3.7 Pulse train created with air throttle manipulation . . . . . . . 44 
3.8 Flowchart of system identification process . . . . . . . . . . 45

3.9 Output data generated for system identification example (0-25 sec: generation, 25-50 sec: validation) . . . . . . . . . 48

$3.10 \epsilon=0$, linear model structure . . . . . . . . . . . . . . . . . . 49

$3.11 \epsilon=0.2$, linear model structure . . . . . . . . . . . . . . . . 49

$3.12 \epsilon=0.2$, nonlinear model structure . . . . . . . . . . . . 50

3.13 Flowchart of unstart specific system identification . . . . . . . 51

3.14 Illustration of transducer location effect . . . . . . . . . . . 53

3.15 Block diagram of Hammerstein-Wiener model structure . . . . 55

3.16 Model generation run, flap constant with VGJ pulses . . . . . 58

3.17 Model generation run, flap pulses with VGJs constant . . . . . 58

3.18 Model validation run, variation in both flap and VGJs . . . . 59

3.19 First model generation run . . . . . . . . . . . . . . . . 61

3.20 Second model generation run . . . . . . . . . . . . . . 61

3.21 Model validation run . . . . . . . . . . . . . . . . . 62

4.1 Simplified model block structure . . . . . . . . . . . . . 66

4.2 Quadratic fit describing equivalence ratio vs. shock location relationship, $R^{2}=0.992 \ldots \ldots$. . . . . . . . 71

4.3 Exponential fit describing equivalence ratio and air throttle vs. $X_{\Delta}, R^{2}=0.759 \ldots \ldots \ldots \ldots \ldots$

4.4 Linear fit describing distance from equivalence ratio-based shock to selected transducer and air throttle vs. pressure output scaling, $R^{2}=0.887 \ldots \ldots \ldots \ldots$. . . . . . . . . . . 73

4.5 First model generation run . . . . . . . . . . . . . . . . 74

4.6 Second model generation run . . . . . . . . . . . . . . . . . . . 74

4.7 Model validation run . . . . . . . . . . . . . . . . 75

4.8 Model validation run, second transducer location . . . . . . . 76 


\section{Chapter 1}

\section{Introduction}

\subsection{Overview}

Air-breathing engines capable of high-speed flight have been fixture of aerospace research for many years. Engines capable of supersonic or even hypersonic velocities offer potential solutions for rapid-travel vehicles and weapons as well as integration of air and space operations [1]. Scramjet engines in particular have been pursued as a means to accomplish this feat as they theoretically permit operation much further into the range of hypersonic velocities (above Mach 6) than other supersonic vehicles such as turbojets (below Mach 3) and ramjets (Mach 3 to 6) [2]. A scramjet functions with no moving parts, instead using the geometry of the inlet to bring incoming air to conditions favorable for combustion. This is accomplished through multiple steps of compression and deceleration largely achieved through a complex series of oblique and normal shock structures that are present in the inlet and isolator [2]. Unlike the ramjets, the flow is not decelerated to subsonic speeds but instead undergoes combustion at a supersonic velocity. This is especially desirable at high Mach numbers for reasons such as avoidance of fuel dissociation [2]. However, particularly useful are dual-mode engines that can switch between operation as a ramjet and operation as a scramjet. They offer the 
additional benefit of increased operational range covering supersonic to highly hypersonic speeds [3]. Much of the work contained in this thesis is based on hardware models that simulate conditions encountered by a dual-mode engine.

Although the potential benefits of supersonic and hypersonic propulsion are clear, the technology is not without problems. Inlet unstart is a harmful change in flow condition that can occur during high-speed flight causing a significant reduction in mass flow to the inlet. Unstart ultimately leads to loss of thrust, engine failure, and possible structural damage [2]. Unstart can be brought on by multiple types of disturbances, however this work will focus on the case where an increase in engine back pressure pushes the leading edge of the shock structure in the inlet back towards the throat [4]. At a certain point the leading edge of the shock, known hereafter simply as "the shock", will be ejected of from the inlet and will suddenly return the flow to pre-started conditions [5].

Clearly active control of the shock system is necessary. Methods for avoiding unstart however have been slow to develop due to the inherent dynamic complexity of the problem. Most notably, the onset of unstart can be very fast - sometimes on the order of milliseconds for hypersonic velocities. Algorithms that use current measurements to check for events at previous time steps cannot be reliably used to monitor incoming data for changes signaling the onset of unstart as this may lead to prohibitively late detection and control decision. Instead, viable options for unstart detection are algorithms that make use of change thresholds. Generally, the change-threshold based algo- 
rithms cannot be implemented over a wide range of operating conditions essentially due to the fact that the underlying threshold must be manually adjusted as changes to flight conditions occur. Another challenge impacting feedback control solutions for unstart suppression is the lack of a tractable mathematical model to represent the transient dynamics of shock motion. Such a model would ideally be derived from the full dynamics of flow within the engine - a process that would be prohibitively difficult if not entirely impossible for a hypersonic vehicle.

\subsection{Related Work}

As unstart can have detrimental effects on engine operation, significant effort has been made in recent years to determine ways to monitor and control conditions leading to its onset. Experimental efforts towards unstart control typically feature simplified engines that are outfitted with high-speed sensors. These sensors can collect information that will display abrupt changes that correspond to or preempt the sudden operational shift between started and unstarted flows. Pressure data in particular is a relatively simple metric to monitor that has been shown to accompany shock motion leading to unstart $[6],[7],[8]$. Other types of data such as temperature [9] or water vapor and $\mathrm{OH}$ content [10] have been investigated but are often more difficult to obtain and as of yet do not provide either significant or consistent indication of unstart in advance of pressure data. Information from pressure transducers throughout the engine has been manipulated in various ways in an attempt to 
extract metrics that can be monitored for changes that indicate the advancing shock structure. Le et al. [11] and others [12], [13] for example make use of magnitude, standard deviation and power spectrum techniques that lend themselves to simple thresholding. Trapier et al. [14] look at additional frequency techniques such as spectrograms, wavelet transforms, and WignerVille distributions that are specifically designed to highlight time-evolving characteristics of the data. They also present a variety of existing change detection algorithms adapted for identifying abrupt changes in the resulting information. Tan et al. [15] also present time-frequency techniques that make use of pressure data but suggest that pattern recognition algorithms are more suited to unstart detection.

Some attempts to implement control schemes in hardware have been made, but the rapid onset of unstart makes it difficult to detect the event and actuate in time to prevent it. Efforts are still focused on developing actuation methods that can respond effectively in a high-speed flow and on a time scale appropriate to the problem. The use of Wheeler Doublets and Vortex Generator Jets in particular have been investigated in [16] and are further discussed in this work. Recently, plasma based actuation techniques have also been applied to supersonic flows [17].

Model development is also becoming a more prevalent branch of unstart investigations. Several computational fluid dynamics techniques have recently been adapted or developed with the intent to represent the supersonic conditions [18], though this work focuses primarily on generating flow simulations 
that match experimental data. However, some have used these simulations to help develop mathematical descriptions for unstart. Tao et al. [19] for example have used catastrophe theory to define limits on operating conditions that maintain started flow. Others have used experimental data to fit established model structures with system identification on a case by case basis [20].

\subsection{Contributions of Thesis}

In this thesis both of the aforementioned challenges concerning unstart detection and transient dynamics representation are addressed. In Chapter 2, the unstart detection problem is undertaken by investigating a wide array of automated change detection algorithms that can be used upon incoming pressure data at various sensing locations to determine when the leading edge of the inlet shock structure passes by the transducer. The modeling problem is addressed next by directly using experimental data to aid development of a transient dynamics model for shock motion. In Chapter 3 system identification techniques are used to investigate model structures that are most suitable

for reliable prediction of pressure data in response to various engine inputs. Analysis of the system identification models however makes clear an important observation: the input-output behavior of the pressure output is largely linear, save a few identifiable and describable sources of nonlinearity. Thus, in Chapter 4 a simplified model is developed that separates and analytically prescribes the nonlinear dynamics while only leaving the linear dynamics to be described by system identification. 


\section{Chapter 2}

\section{Change Detection}

During unstart, the upstream propagation of the shock towards the engine throat can been seen through several types of system measurement data. However, the pressure measurements along the inlet provide a particularly clear view of the shock's location as the pressure suddenly and dramatically rises as the shock passes. A change detection algorithm can monitor the pressure history at a given point and determine when an abrupt pressure rise occurs. If this kind of information can be obtained at points throughout the inlet then a control scheme has an improved basis on which to make actuation decisions and prevent unstart.

Since the timescale of unstart at high Mach numbers can be as small as milliseconds, change detection algorithms that use current data points to check for events at previous time steps are not practical options given realistic sampling frequencies and constraints on processing times. Instead, viable options are algorithms that make use of change thresholds. Typically the thresholds must be set by the user and ultimately means that the algorithm cannot generally be implemented over a wide range of operating conditions without human attention. The goal here is thus to find an algorithm that 
reduces or eliminates the user interaction necessary for successful implementation on any unstart detection problem. An initial user-set threshold might be permissible, especially if it does not need to be changed across different data sets, although an automatically selected threshold would be better. Additionally, an algorithm would need to not just signal onset of unstart but do so in a timely manner so that there is as much time as possible for actuation.

A variety of change detection algorithms have been implemented in this work including standard deviation, power spectrum, and statistical-ratio based methods. Each algorithm was tested on four different sources of highspeed pressure transducer data resulting from experiments at both the Air Force Research Laboratory (AFRL) and The University of Texas at Austin (UT). A successful algorithm would provide a reasonable detection rate across all four sources with minimal to no human involvement. The most promising algorithm, Cumulative Sum detection (CUSUM), is discussed here and in the reference material [12], [14]. Performance of the algorithm is evaluated on four dissimilar data sets and then compared to the performance of other established change detection algorithms.

\subsection{Data Sets}

\section{Data Set 1: AFRL 2008}

The first data set was produced by a continuous-flow, direct-connect, supersonic combustion facility at the Wright-Patterson Air Force Base AFRL location in 2008. The hardware set up for the experiments that generated this 
data set simulated flight conditions around Mach 5. The flowpath had a rectangular cross-section and consisted of a Mach 2.84 facility nozzle, constant 9 in width inlet and isolator, combustor with additional step-shaped cavity, and divergent geometry tailpipe and exhaust adapter. The reference height, defined at the combustor entrance, was $1.664 \mathrm{in.}$

The flowpath is depicted in Figure 2.1. The figure also depicts the multiple groups of flush-mounted fuel injectors that were available. Typically three injector groups were used in a given run. Each injection site had its own dedicated supply of electrically heated gaseous hydrocarbon fuel. All injectors used in the runs presented in this work were oriented perpendicular to the flow. In cases with multiple injector groups being used, the total equivalence ratio selected for the run would be accomplished by distributing a portion of the fuel to each injector group as determined by a user-selected percentage split. Several high-speed pressure transducers, with variable sampling rate, were mounted along the bottom of the isolator. Their locations are shown in Figure 2.1 in inches (non-normalized) from the beginning of the inlet/isolator (symbolically, the engine throat). The transducers were not flush mounted and stood off from the hardware by a significant distance. Lower speed transducers were available on many of the other fuel lines and control valves. This information and further details are discussed in [10].

Data Set 2: AFRL 2011

The experiments for this data set were conducted at the same AFRL directconnect test facility in 2011, however this time with a circular cross-section 


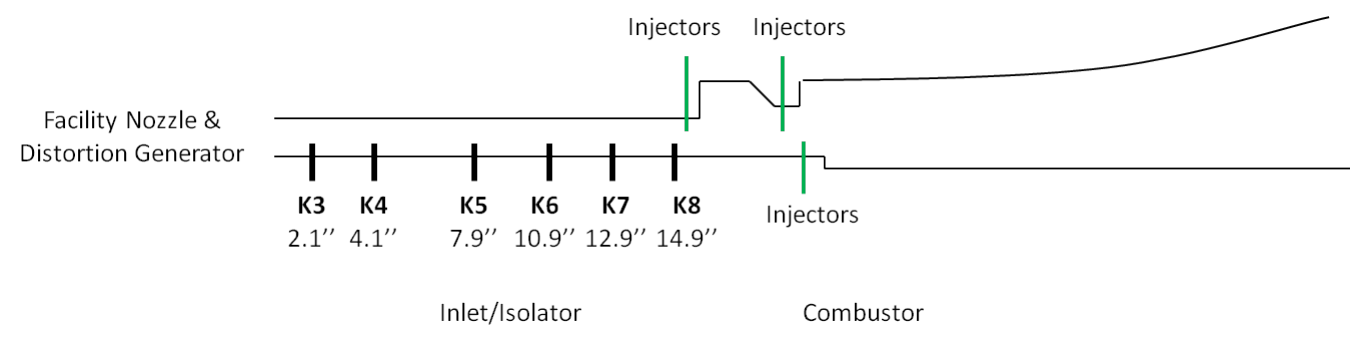

Figure 2.1: Hardware schematic for Data Set 1

flowpath. The experiments simulated flight conditions around Mach 4 with $P_{0}=55$ psia and $T_{0}=1800 \mathrm{R}$. The flowpath consisted a Mach 1.8 facility nozzle, an 12 in long inlet and 24 in long isolator each with $0.25^{\circ}$ divergent geometry, and a divergent geometry combustor, two 16 in divergent geometry tailpipe sections, and an exhaust adapter. Gaseous ethylene fuel was injected flush into the combustor from multiple locations as indicated in Figure 2.2. The equivalence ratio split was again variable and user-specified. High-speed pressure transducers were placed at the locations indicated in the same figure along the isolator at a radial location of $120^{\circ}$. Note that the transducer locations do not cover as much of the isolator as shown in the figure but are rather clustered upstream. The graphic is skewed for clarity. Again the transducers were not flush-mounted though the stand-off distance had been greatly reduced to an average of 1-2 inches. Transducers with lower sampling rates were available on many of the other fuel lines and control valves. These specifics and additional information are provided in [21].

Data Set 3: UT 2008

Data for this set was collected in 2008 from a inlet/isolator model in a blow- 


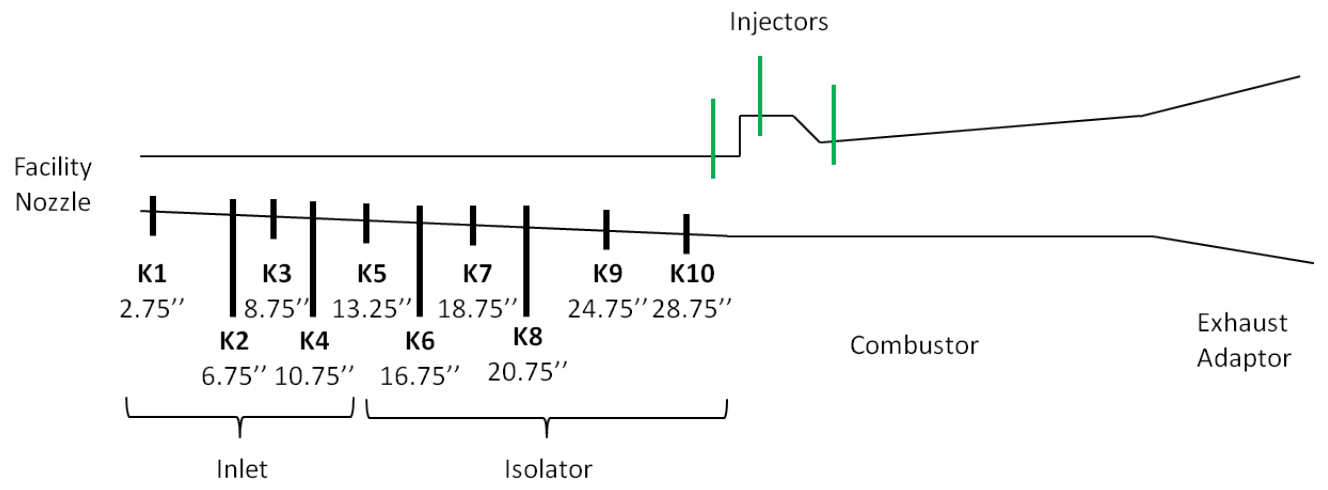

Figure 2.2: Hardware schematic for Data Set 2

down wind tunnel facility in the Flowfield Imaging Laboratory at UT. The experiments were conducted at Mach 5 freestream conditions with an approximate stagnation pressure of 355 psia and stagnation temperature of $575 \mathrm{R}$. The $6^{\circ}$ compression ramp inlet had a height of 1.35 in at the entrance and sidewalls swept back at $53.5^{\circ}$ with respect to the y-axis. The constant-area, rectangular geometry isolator had a width of 6 in, height of 7 in, and length of 30 in. Change in back pressure in this non-reacting flow was simulated by raising and lowering a mechanically-driven flap at the back of the model, as shown in Figure 2.3. High-speed pressure transducers were flush mounted and placed along the isolator and inlet either at or near the center of the bottom wall. Locations are are also given in the figure where distances are normalized by the isolator height ( $h=1$ in) and measured from the entrance of the inlet. The upstream edge of the flap was fixed at the normalized distance of $x / h=12.9$. This and further information can be found in [12]. 


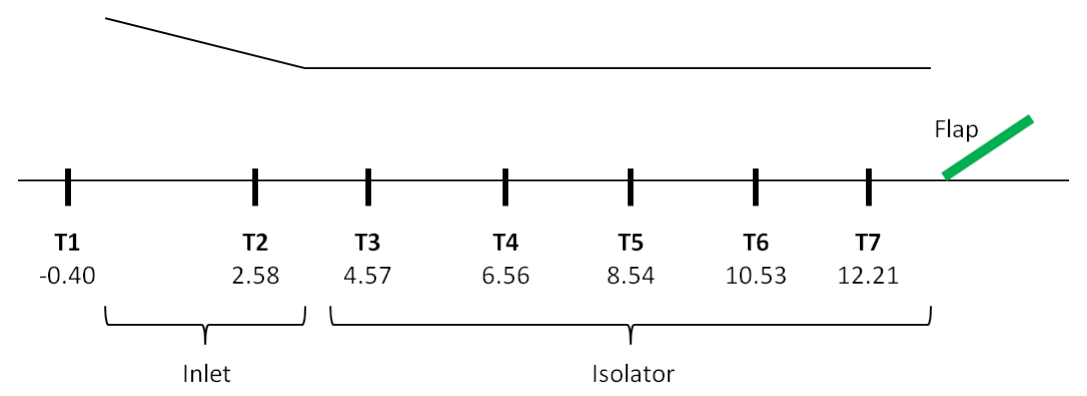

Figure 2.3: Hardware schematic for Data Set 3

Data Set 4: UT 2011

Data for the final set was collected in 2011 from a hardware set up similar to that in Data Set 3. The same inlet/isolator and flap model was used, although here with Mach 2 freestream conditions. Additional pressure transducers were added in between some of the existing transducers as shown in Figure 2.4. Again distances are given as normalized by the isolator height. Pulsed and vortex generator jets were added to the side walls of the model and used in some of the runs to attempt to actively alter the unstart process. However, only runs without jets are used in the change detection work presented here so as to permit fair comparison with the other data sets. Data for the system identification work will make use of the runs with the jets, and their usage will be further discussed with the system identification material.

\subsection{CUSUM Algorithm}

The CUSUM algorithm has long existed in the field of statistical monitoring and quality control. It was first introduced by Page [22] as a means of 


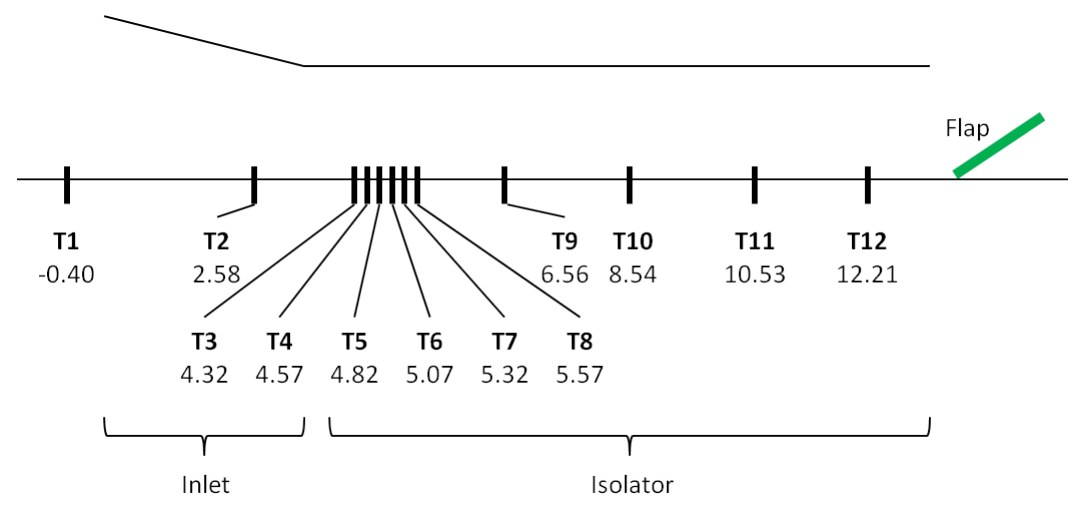

Figure 2.4: Hardware schematic for Data Set 4

monitoring production processes in an industrial setting. A simplified, more generic version was later presented [23], and the algorithm has since found application in many fields where automated detection of change is desired. Numerous variants of the algorithm have been made to address many specific needs such as those arising from noisy data or a particular probability distribution. Today CUSUM applications range from image processing [24] to economics [25] and retrospective analysis in the medical field [26].

The CUSUM algorithm in the context of unstart detection operates by detecting a change in the probability density of the pressure signal. Before the shock passes a pressure transducer, the pressure signal at that point will be more closely aligned with a signal of probability density $p_{0}(x)$. After it passes the pressure signal will be more similar to a signal of probability density $p_{1}(x)$. A change from $p_{0}(x)$ to $p_{1}(x)$ can be detected by monitoring the evolution of the log-likelihood ratio, $s_{i}$, of these densities:

$$
s_{i}=\ln \frac{p_{1}\left(x_{i}\right)}{p_{0}\left(x_{i}\right)}
$$


Here $x_{i}$ is the pressure measurement at time step $i$. As long as the pressure signal is closer to $p_{0}(x), s_{i}$ will be negative. Conversely, $s_{i}$ will be positive when the signal is closer to $p_{1}(x)$. The cumulative sum of the ratios up to the current time step $k, S_{k}$, will thus continue to decrease until the change occurs. After the change $S_{k}$ will begin to increase. A threshold condition can be placed on this increase by comparing the current value of $S_{k}$ to its minimum value $m_{k}$. Once the difference between $S_{k}$ and $m_{k}$ is larger than a user-defined threshold, $h$, an alarm is given.

$$
\begin{gathered}
m_{k}=\min _{1 \leq j \leq k} S_{j} \\
t_{\text {alarm }}=\min \left(k \mid S_{k}-m_{k} \geq h\right)
\end{gathered}
$$

The pressure signal however requires some pretreatment in order for the algorithm to function most effectively. First, the running average of the original pressure signal should be subtracted from the current data point to create a zero-mean signal. Next, the signal should be run through a low-pass filter to reduce noise effects. Unstart pressure data however appears to give earlier detection without filtering more often than with filtering. This effect is briefly visited later on in Section 2.4.1. Finally, an average of the treated pressure signal over a window of the last few seconds should be subtracted from the current data point. This step rarely affects the signal before the change occurs but ensures that that the slight increase occasionally seen when the shock is sitting just behind a transducer does not trigger a false alarm. All of these preprocessing steps can be implemented online. 
The algorithm also requires that $p_{0}(x)$ and $p_{1}(x)$ be known. This can be remedied by assuming $p_{0}(x)$ to adhere to a Gaussian distribution. A Gaussian density still however requires knowledge of the standard deviation and mean of the signal, but these parameters can be calculated from the acquired data. The standard deviation is best found from calculating the standard deviation of the data in a small window just prior to the current point. The mean, ignoring the possible trend subtraction at the end of preprocessing, is zero. We also assume $p_{1}(x)$ to be Gaussian. Here we maintain the mean is anticipated to be zero, but the standard deviation for $p_{1}(x)$ cannot be calculated. Instead, we simply define the standard deviation after the change to be the standard deviation before the change multiplied by a scaling gain $K$. The larger $K$ is, the larger the statistical change must be before the signal begins to align with $p_{1}(x)$. Again, the steps of the CUSUM algorithm can be implemented online. A flowchart describing the CUSUM algorithm is shown in Figure 2.5.

Figure 2.6 provides an example of CUSUM application by running the algorithm on a selected transducer in Data Set 1 . Here, the window for standard deviation calculation was set to $0.5 \mathrm{sec}$ and the window for trend subtraction to 1 sec. The standard deviation gain scaling was set to $K=2$. Finally, the $S_{k}$ rise threshold was set to $h=125$. With these settings the alarm was given at $7.8404 \mathrm{sec}$. The CUSUM algorithm is suited to work across multiple data sets as it reduces data to a simple metric that requires only a single threshold. Unlike other algorithms the threshold is defined in terms of a relative change in the metric, and thus does not require that the metric produced 


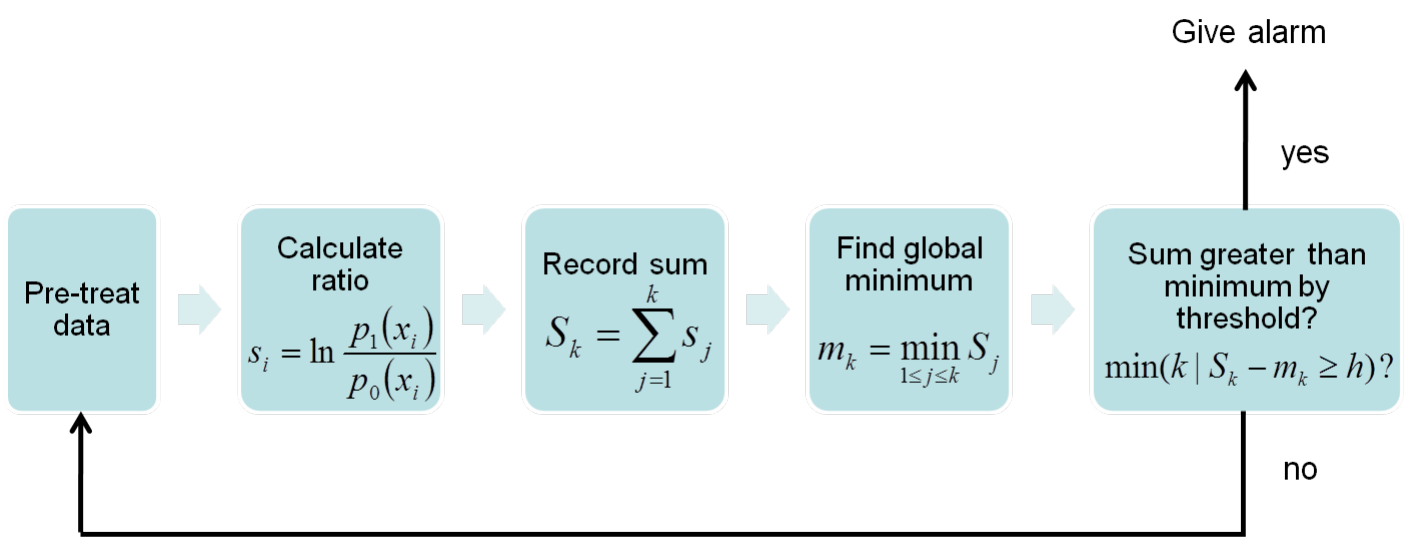

Figure 2.5: Flowchart of CUSUM algorithm

by every data set be of the same magnitude. Application of the same parameters to other transducers, runs, and data sets later shown in in Section 2.4.2 illustrates that no adjustment is necessary for acceptable detection rates. Motivation for the selection of algorithm parameters will also be discussed here. 

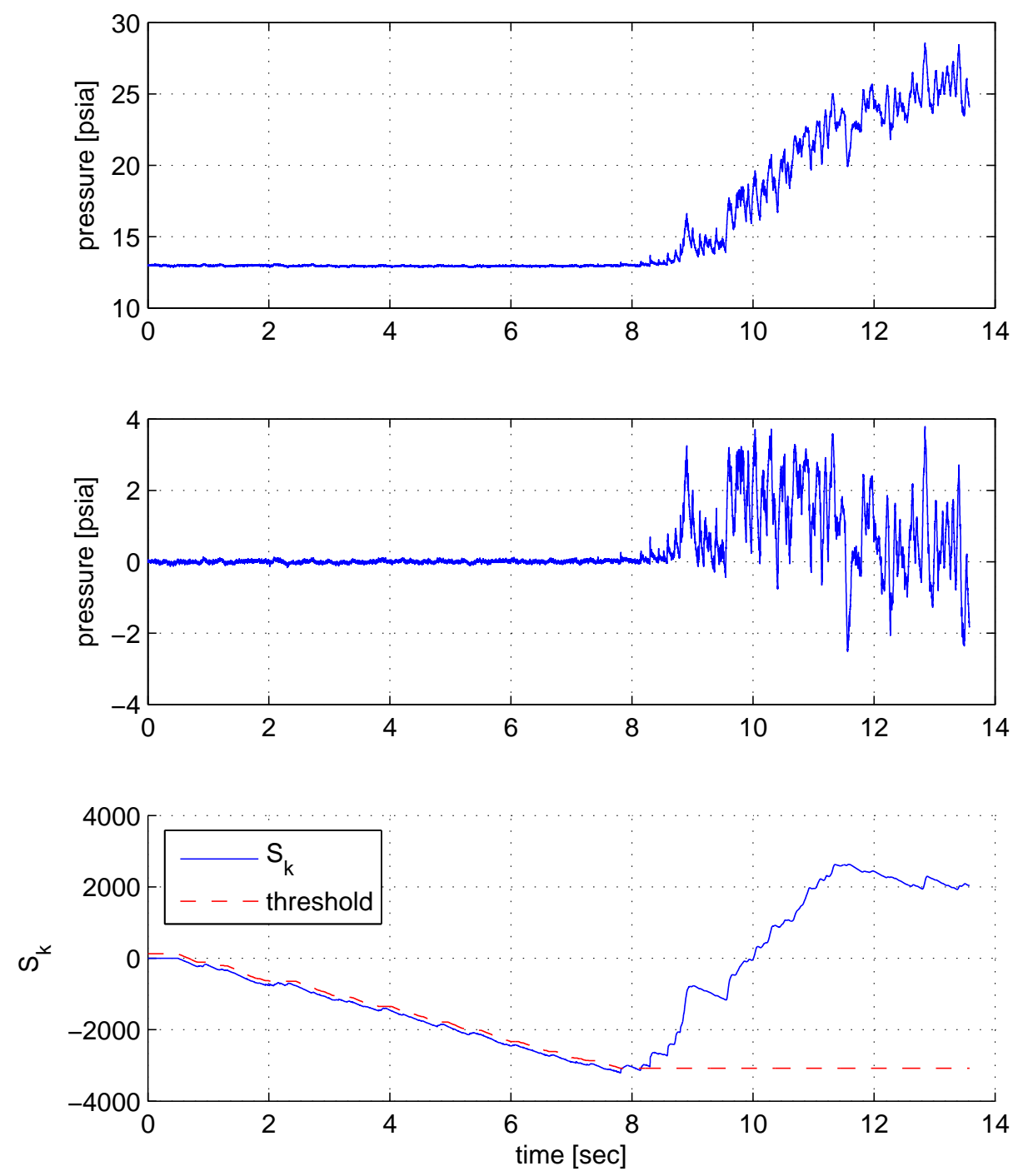

Figure 2.6: Top: Original pressure signal Middle: Processed pressure signal Bottom: CUSUM metric and threshold 


\subsection{Other Algorithms}

\subsubsection{Power Spectrum Based Detection}

In [12], a change detection algorithm is presented that is based on pressure data's spectral power peak seen in the $300-400 \mathrm{~Hz}$ band around the time of unstart. The data set used in [12] is also one of the four data sets used in this work. Ideally, the characteristic peak could be used to develop a detection scheme that detects the beginning of unstart in a pressure signal arising from any arbitrary inlet geometry. However, the presence of this 300-400 Hz peak is not confirmed across a variety of geometries and flow conditions. Thus, before proceeding with application of a similar change detection algorithm it is prudent to check the spectral content of pressure signals in the four available data sets to determine whether they contain a similar peak.

First consider a pressure signal from the data used in [12], known here as Data Set 3. Figure 2.7 illustrates the spectral content of the signal in a timespan prior to the onset of unstart in the top graph and during/after its onset in the bottom graph. A clear peak develops in the 300-400 Hz region. As a counter example, Figure 2.8 shows the spectral content of a pressure signal from Data Set 1. It is clearly seen that that the $300-400 \mathrm{~Hz}$ peak is not seen in this case. In fact, out of the four data sets the $300-400 \mathrm{~Hz}$ peak is only present in Data Set 3. Although the $300-400 \mathrm{~Hz}$ peak is not universally seen, a given data set usually contains its own characteristic peak. The band associated with the peak varies across data sets (and sometimes even transducers), and one data set did not exhibit any peak around the time of the shock's passage. All 

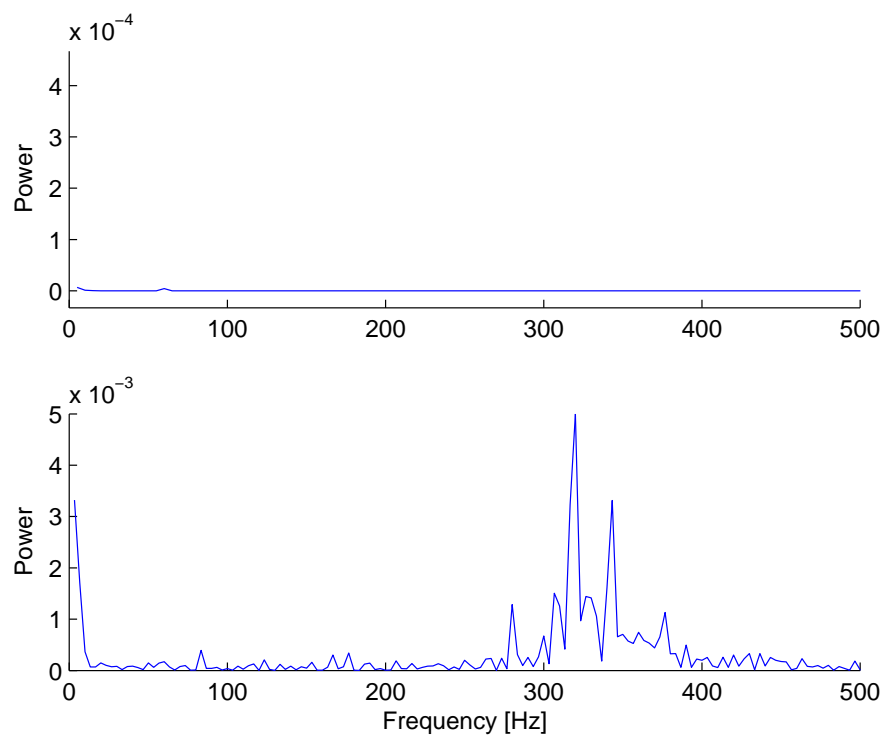

Figure 2.7: Top: Power spectrum of pre-change signal Bottom: Power spectrum of signal during and after shock's pass exhibiting peak

four sets do however display a notable signal amplification in the low frequency range. This is somewhat expected as the shock passes over the transducer, but the trend appears to persist (to a lesser extent) once the shock has completely passed. We therefore proceed by adjusting the algorithm to monitor changes in the $1-15 \mathrm{~Hz}$ band instead of the $300-400 \mathrm{~Hz}$ band. Note that monitoring the low frequency growth does not offer the benefit of early detection as this growth is directly associated with the shock passing over the transducer. Growth in a higher frequency band, should any have been seen, would be more likely to be associated with other factors related to the approaching shock. Should those factors precede the approaching shock then monitoring the associated frequency band would carry the potential of early detection. 

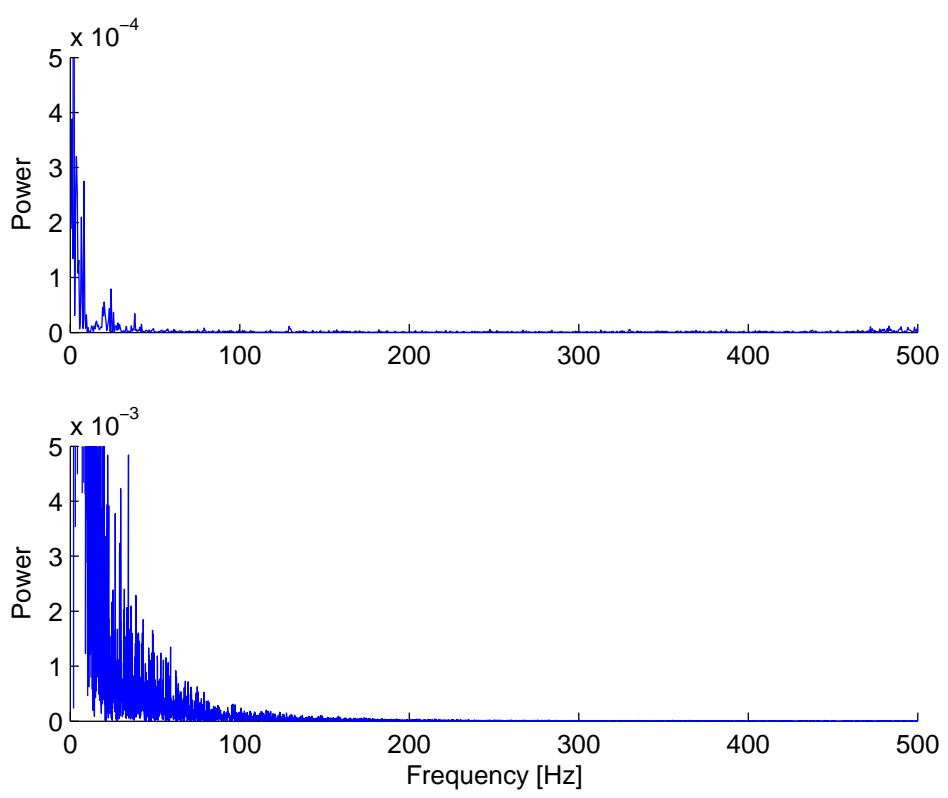

Figure 2.8: Top: Power spectrum of pre-change signal Bottom: Power spectrum of signal during and after shock's pass lacking peak

The algorithm used here is very similar to that presented in [12]. A discrete short-time Fourier transform is employed where the current pressure data point is taken along with a window of prior pressure data and then used in an FFT-based calculation to determine the spectral power associated with that selection of information. The power in the frequency band of interest is then averaged, thus producing a single data point for the window. When the next pressure measurement becomes available the window shifts one time step and the process is repeated. Note that resolution of the short-time Fourier transform output can be very coarse if few data points are used at each step. This can be improved by padding the signal with content outside the frequency band of interest. If the frequency band selected for monitoring consistently 
contains a peak in the power associated with the passing shock, then the metric produced should display a more pronounced change at that time. This will make the change easier to detect. The user must initially set a threshold condition for this metric to exceed. In this implementation the maximum value of the metric over a baseline initialization time window is determined and then multiplied by a user-selected scaling factor. The result is the threshold for

the rest of the run. A persistence criterion requiring multiple data points to exceed the threshold before the alarm is given is necessary with this algorithm as pressure spikes in the pre-change region will certainly be amplified. Figure 2.9 illustrates the $1-15 \mathrm{~Hz}$ power spectrum algorithm running on the same sample run used previously. At each step the Fourier transform uses a window of approximately $0.2 \mathrm{sec}$ of points (data and padding). Following the example of implementation in [12], the first $40 \%$ of this window is actual data (250 points or $0.08 \mathrm{sec}$ in this example) and the remainder is padding (375 points or $0.12 \mathrm{sec})$. The threshold is calculated by averaging the metric over the first $0.5 \mathrm{sec}$ of data and then scaling by 1.5. A persistence criterion requiring 2 out of the last 3 data points to exceed the threshold must be satisfied before an alarm signaling the approaching shock is given. The alarm is given at 7.8164 sec.

\subsubsection{Standard Deviation Based Detection}

Monitoring the standard deviation of the pressure signal is a commonly used, simpler means of change detection [7]. In this method a constant thresh- 

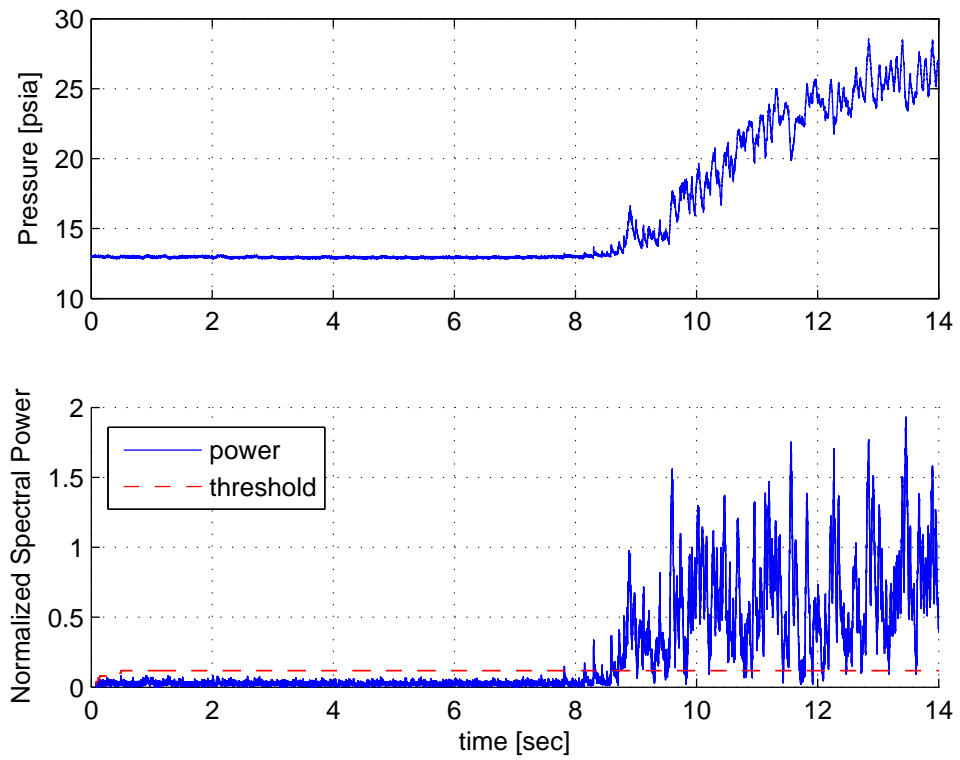

Figure 2.9: Top: Original pressure signal Bottom: Normalized spectral power and threshold

old is set, and the standard deviation of the pressure signal over a specified window of time (ending at the current data point) is calculated. The alarm is given once the standard deviation exceeds the threshold. Note that for a quite pre-change signal the standard deviation will be near zero. It is thus ineffective to set the threshold as a scaled value of the standard deviation and instead a constant value must simply be selected. The window size selected significantly contributed to the smoothness of the signal produced after the standard deviation calculation. A larger window size masks noise and occasional pressure spikes but may delay detection. A smaller window size may offer earlier detection but at the risk of false alarms due to failure to reject the less mediated spikes. A persistence criterion requiring several points in addition to the 
current data point to have crossed the threshold will help in this case. The standard deviation algorithm offers potential improvement over the pressure magnitude algorithm as it levels the incoming data to some degree and makes the successful use of a universal threshold more likely. However, use of the algorithm has shown that while one threshold setting may be appropriate for all the transducers and runs in one data set, the threshold typically requires adjustment when switching to another another data set. Figure 2.10 shows the algorithm applied to the sample run. The standard deviation threshold here has been set to 0.1 . A window size of 800 samples, or approximately $0.256 \mathrm{sec}$ in this example, has been used to calculate the standard deviation associated with an incoming data point. Again, no persistence criterion is used here. The alarm is given at 8.3021 sec.

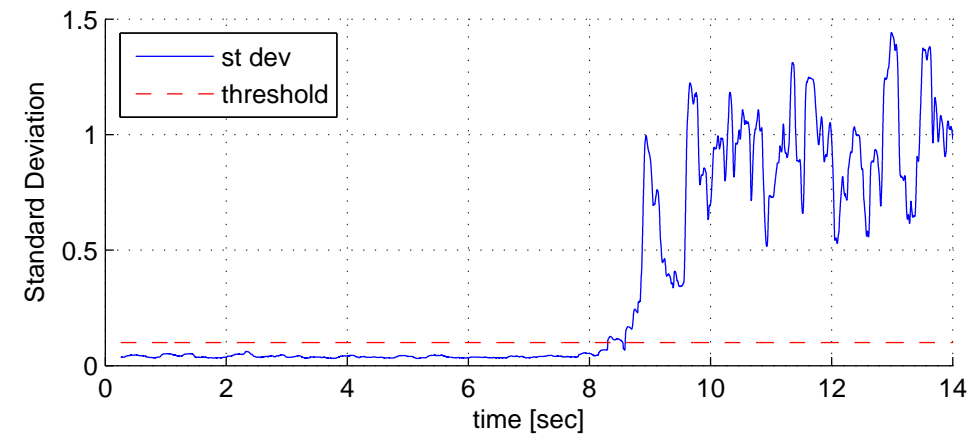

Figure 2.10: Standard deviation of pressure signal with threshold

\subsubsection{Pressure Magnitude Based Detection}

The simplest possible form of change detection is to directly monitor the magnitude of the pressure signal itself. With this method a single, constant 
threshold is set and an alarm is given as soon as the pressure signal rises above the threshold. Although simple, this method is also algorithmically crude and will likely require extensive threshold adjustment when applied across various transducers or runs. A persistence criterion requiring a certain percentage of the immediately preceding data points to exceed the threshold in addition to the current data point may also be necessary, especially when the threshold is tightly set. Figure 2.11 shows such an algorithm applied to the sample run. Here the threshold has been set by calculating the average pressure over the first 0.5 seconds of the pre-change signal and scaling it by value of 1.05 . The alarm is given at $8.2999 \mathrm{sec}$. No persistence criterion is used in this example.

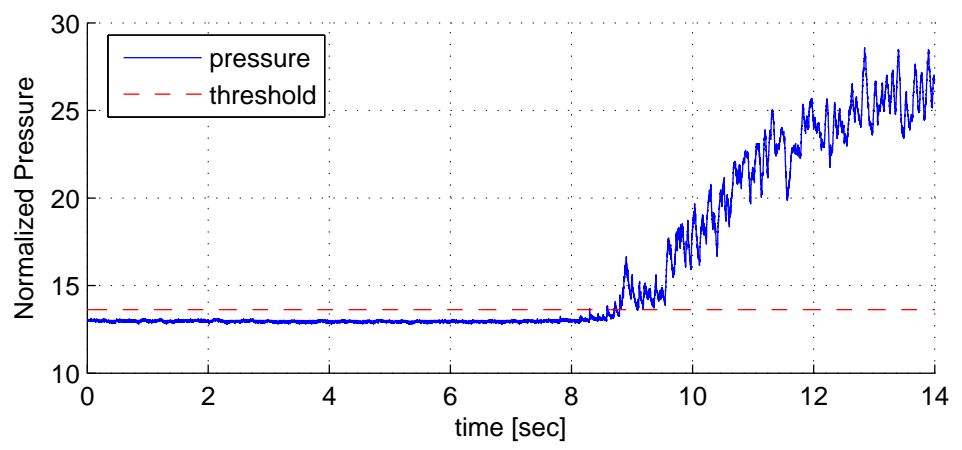

Figure 2.11: Pressure signal with threshold

\subsection{Algorithm Performance}

This section will investigate the appropriateness of the CUSUM algorithm for reliable unstart detection across multiple sources of data. A comparison of detection times for filtered and unfiltered data is presented first in 
order justify the the choice to use unfiltered pressure signals. The feasibility of using a universal set of settings in the CUSUM algorithm to indicate unstart is presented next by illustrating that the same settings can be used successfully determine the time that the shock passes a given transducer in each of the four data sets. An investigation of the timeliness of detection by the universal CUSUM versus other change detection algorithms is presented last.

\subsubsection{Filtering Comparison}

As previously mentioned, the CUSUM algorithm would typically benefit from data that has been low-pass filtered to reduce the effects of noise. However, unstart pressure data often earlier detection when the data is left unfiltered. Table 2.1 compares the CUSUM detection times of filtered and unfiltered pressure signals in each of the data sets. In the filtered case, the data is passed through a low-pass filter with at cutoff frequency of $100 \mathrm{~Hz}$ (similar in magnitude to the cutoff frequency used in [14]). All algorithm pa-

rameters, thresholds, and other data pretreatments are left the same. The detection times indicate that the unfiltered data provides earlier detection in a little more than half of the cases considered. While the reasons for this are unclear, all further pressure signals used with the CUSUM and other change detection algorithms will be left unfiltered. 
Table 2.1: Comparison of detection times for unfiltered data and low-pass filtered data (100 Hz cutoff)

\begin{tabular}{|c|cc|}
\hline Transducer & CUSUM-unfilterd & CUSUM-filtered \\
\hline \multicolumn{3}{|c|}{ Data Set 1 Detection Times [s] } \\
\hline K5 & 7.8404 & false alarm \\
K6 & 7.3928 & 7.2840 \\
K8 & 6.3215 & 6.1794 \\
\hline \multicolumn{4}{|c|}{ Data Set 2 Detection Times [s] } \\
\hline K2 & 62.3523 & 62.2357 \\
K3 & 62.4046 & 62.7541 \\
K4 & 61.9410 & 61.9435 \\
\hline \multicolumn{4}{|c|}{ Data Set 3 Detection Times [s] } \\
\hline T2 & 1.1683 & 1.1696 \\
T3 & 1.1618 & 1.1631 \\
\hline \multicolumn{4}{|c|}{ Data Set 4 Detection Times [s] } \\
\hline T5 & 0.3590 & 0.3602 \\
T6 & 0.5048 & 0.4580 \\
\hline
\end{tabular}

\subsubsection{CUSUM Application across Data Sets}

In order for the CUSUM algorithm to be useful in an unmoderated, realistic environment it must be able to perform over a wide range of conditions without requiring human intervention. This means that that the algorithm settings must provide acceptable detection for more than just a single hardware set up or flow conditions. Although the ability of the CUSUM algorithm to detect changes is primarily influenced by selection of the threshold, there are a number of other parameters that influence detection to a lesser extent. The lesser parameters can largely be determined through knowledge of the type of abrupt changes typically seen in the unstart problem. After, the selection of the threshold becomes a simple trial and error exercise that quickly reveals the 
a range of successful threshold values due to the same order metric produced by the algorithm regardless of specific pressure signal provided.

The first, and most significant, of the lesser parameters is the size of the sliding window used for standard deviation calculation. This parameter is common to many change detection algorithms and is generally recommended to be set at some value much larger than the range of the event to detect. In the unstart problem the change between pre- and post-shock behavior is very abrupt and usually occurs over a matter of milliseconds. Thus a value on the order seconds would certainly be acceptable, and a window size of 0.5 sec is selected. Next, the size of the window for trend subtraction should be set. As mentioned previously, this parameter allows for any slowly developing trends in the mean of the pre-change signal to be removed. The size of the window should thus be notably larger than the size of the standard deviation window and is set to 1 sec. The last of the lesser parameters is the post-change standard deviation scaling, $K$. In most CUSUM applications this parameter carries a significant amount of weight, however in the context of the often clean and abrupt changes seen with the unstart problem, its selection becomes less important. Most values (restricted to $K>1$ ) will produce acceptable results, though values too close to $K=1$ will over emphasize smaller pressure spikes and variations and may effect detection. The value $K=2$ is selected here. Finally, a threshold value, $h$, can be selected by a brief application of the lesser parameters to a variety of runs and determining when the first major shift occurs in the CUSUM metric and what threshold values would 
be necessary for that shift to trigger an alarm. Carrying out this process on several sample runs from each of the data sets indicates that a threshold value in the range of $100-200$ is appropriate. The value $h=125$ is chosen to air on the side of early detection while still leaving some room to accommodate small spikes in the metric.

Several other notes regarding the application of the CUSUM algorithm to the unstart problem should be made here. Because of the standard deviation of the signal that is used in the CUSUM metric is calculated over a 0.5 second window, there will be no metric available during the first 0.5 seconds of signal monitoring. Hopefully the shock will not pass the transducer in question during this time, but the window can be shortened if this lead time is problematic. As an example, the runs in Data Set 4 are frequently very short (approximately $1 \mathrm{sec}$ total) and the change in the signal often happens before the $0.5 \mathrm{sec}$ mark. For this data set the window size was reduced to $0.25 \mathrm{sec}$ and the CUSUM algorithm and all other previously stated parameter values were implemented without issue. Should the window size change more drastically however, parameter adjustments will likely be necessary. It is also helpful if the sampling frequency for all the runs that attempt to use a set of parameters to have similar sampling frequencies. If the sampling frequency varies too widely there will be some runs with more (or less) data points that comprise pre-change pressure spikes. This will alter the behavior of the metric by giving it more (or less) data to attribute to a statistical shift and thus impact the range of appropriate threshold values. Each of the data sets presented were 
sampled at widely varying rates but all were downsampled to a rate between 1.5 and $1.7 \mathrm{kHz}$ as this range will accommodate millisecond order changes without increasing computational time because of additional data.

Figures 2.12 to 2.14 illustrate CUSUM performance with the described universal settings on one sample from Data Sets 2-4. An example of the performance of these settings on Data Set 1 has already been provided in Figure 2.6. In each figure the original pressure signal in shown in the top graph, the preprocessed signal in the middle, and the CUSUM metric and threshold in the bottom. The change is correctly detected in each case. 

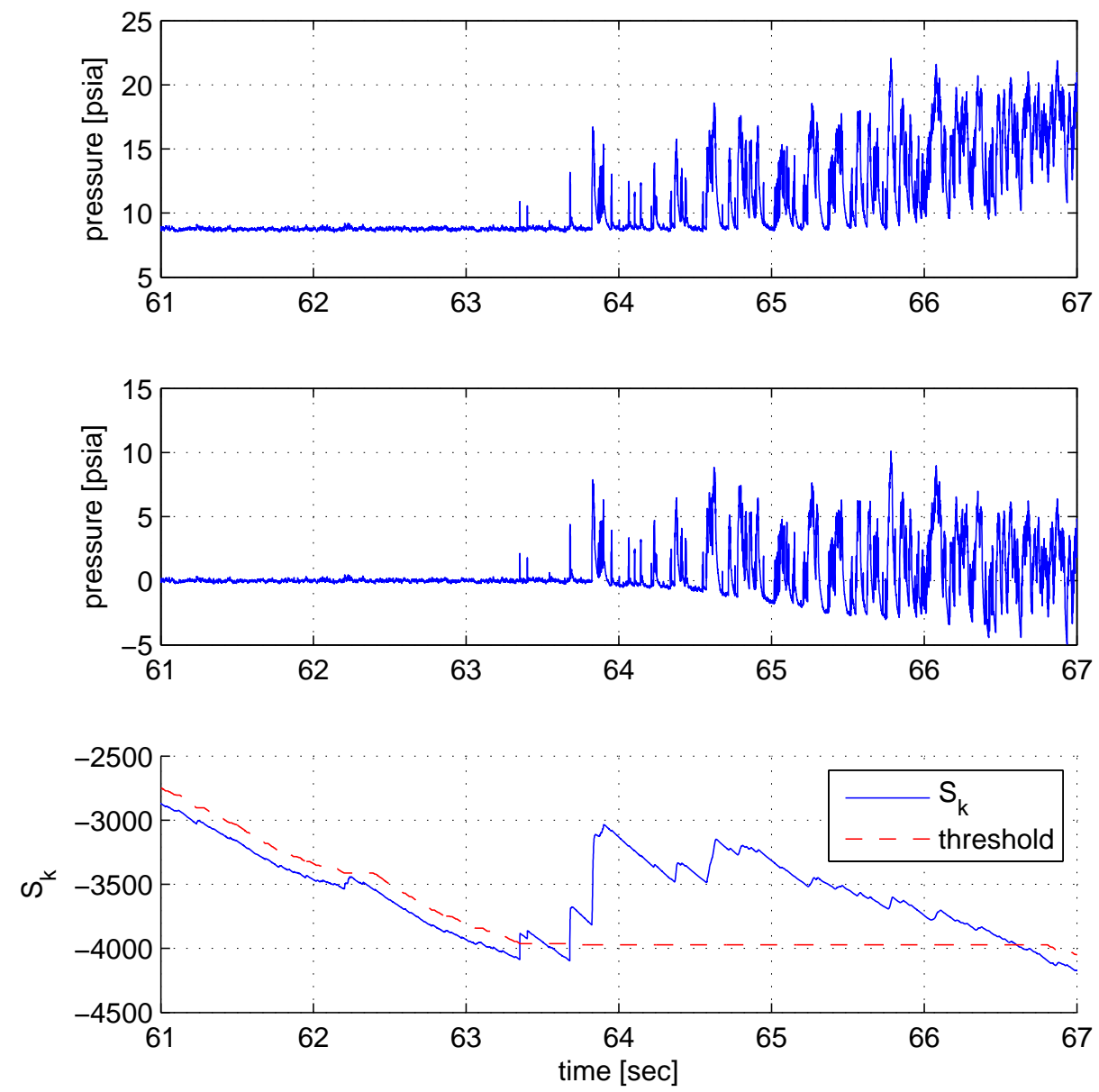

Figure 2.12: CUSUM performance on Data Set 2 example, transducer K2, alarm at $62.3523 \mathrm{sec}$ 

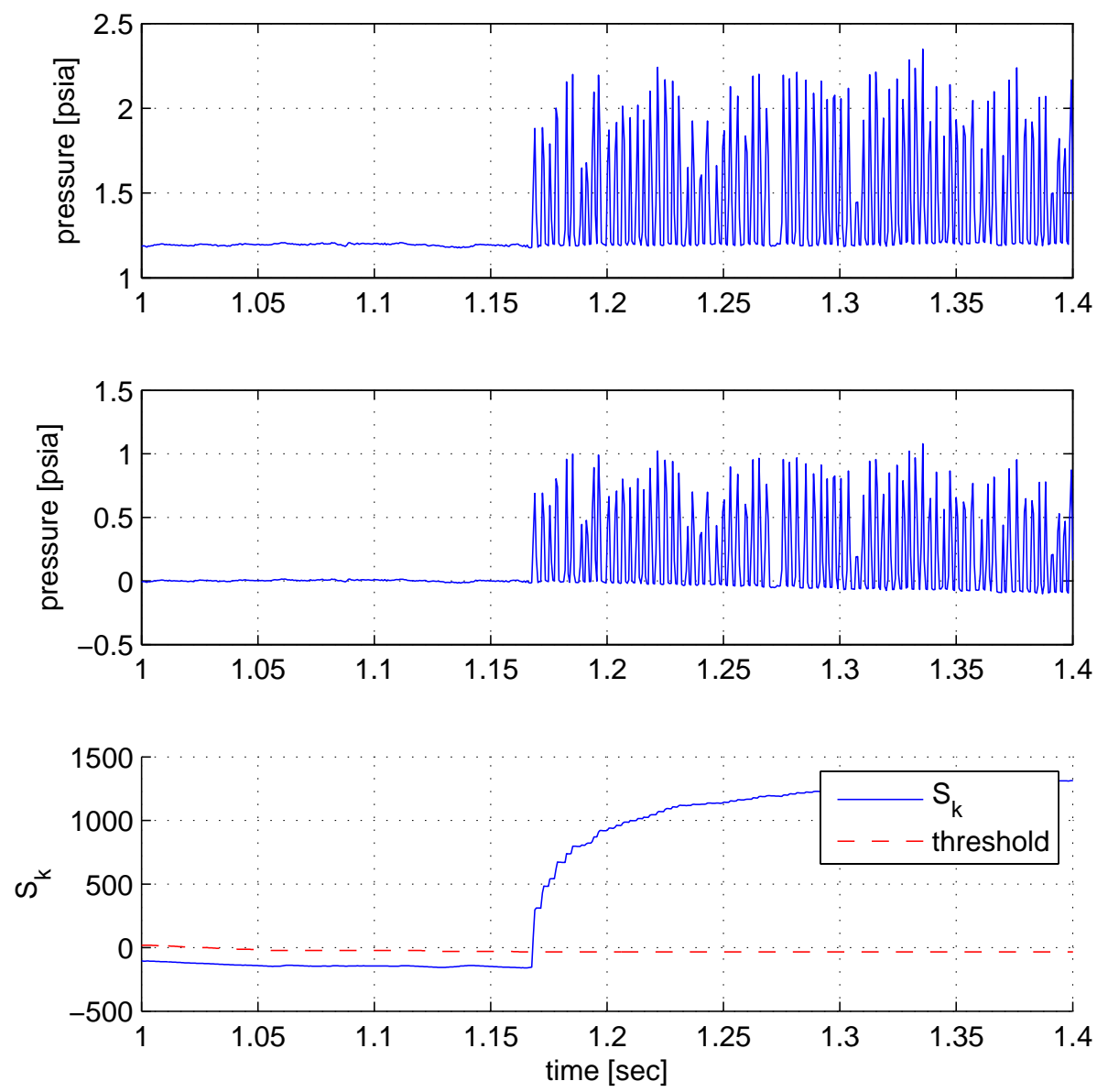

Figure 2.13: CUSUM performance on Data Set 3 example, transducer T2, alarm at $1.1683 \mathrm{sec}$ 

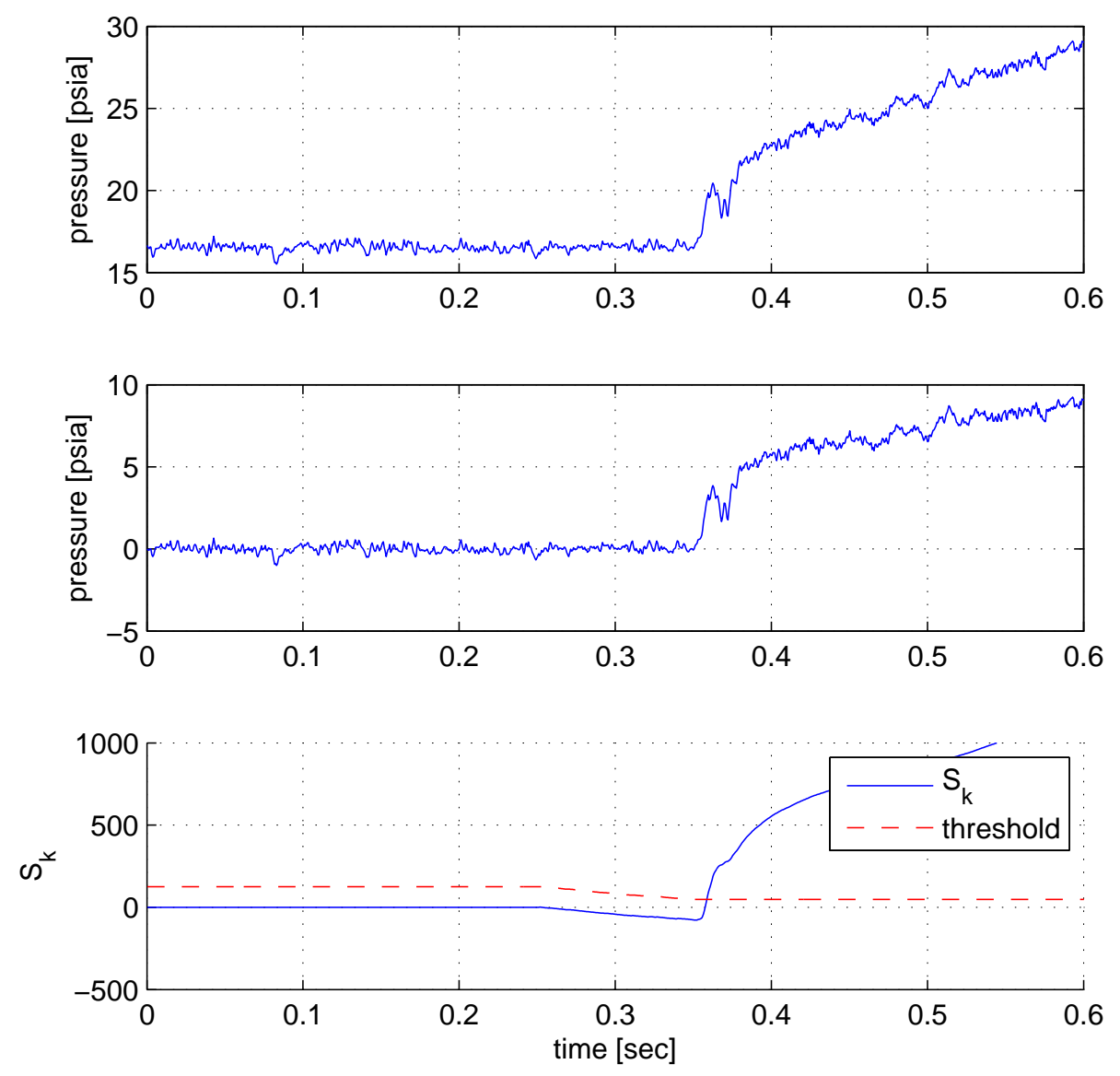

Figure 2.14: CUSUM performance on Data Set 4 example, transducer T5, alarm at $0.3590 \mathrm{sec}$ 


\subsubsection{Multiple Algorithm Comparison}

The performance of the CUSUM algorithm should next be compared to that of other change detection schemes. Table 2.2 reports the alarm times given by each of the algorithms presented earlier when applied to the same sample runs from each data set used in the previous section. The CUSUM algorithm uses the stated universal settings on every run and every transducer. Settings for the other three algorithms are adjusted for each data set (though not for each run or transducer, with the exception of transducers in Data Set 4) so as to provide their best detection times. The universally set CUSUM algorithm typically performs similarly to the specifically set algorithms.

Table 2.2: Comparison of detection times for all algorithms

\begin{tabular}{|c|cccc|}
\hline Transducer & CUSUM & Power & S. Dev. & Mag. \\
\hline \multicolumn{5}{|c|}{ Data Set 1 Detection Times [s] } \\
\hline K5 & 7.8404 & 7.8164 & 8.3021 & 8.2999 \\
K6 & 7.3928 & 7.3936 & 7.3965 & 7.3936 \\
K8 & 6.3215 & 6.4858 & 6.4890 & 6.4855 \\
\hline \multicolumn{5}{|c|}{ Data Set 2 Detection Times [s] } \\
\hline K2 & 62.3523 & 63.3517 & 63.3519 & 63.3513 \\
K3 & 62.4046 & 62.4041 & 62.4045 & 62.4037 \\
K4 & 61.9410 & 61.9413 & 61.9413 & 61.9229 \\
\hline \multicolumn{5}{|c|}{ Data Set 3 Detection Times [s] } \\
\hline T2 & 1.1683 & 1.1696 & 1.1683 & 1.1683 \\
T3 & 1.1618 & 1.1631 & 1.1622 & 1.1621 \\
\hline \multicolumn{5}{|c|}{ Data Set 4 Detection Times [s] } \\
\hline T5 & 0.3590 & 0.3578 & 0.3574 & 0.3554 \\
T6 & 0.5048 & 0.7070 & 0.4346 & 0.4148 \\
\hline
\end{tabular}

The CUSUM algorithm has several features that it handles very well 
compared to the other algorithms. The algorithm has no trouble identifying that a gradual or smooth rise in pressure still indicates a change from the pre-change state. The power spectrum algorithm in particular has trouble identifying this kind of change and will often miss detection all together. The other algorithms potentially have a severely delayed response in this case. Similar circumstances arise when the leading edge of the pressure change displays and indecisive rise, i.e., the pressure does not simply display a rapid increase in magnitude at first. The CUSUM metric typically begins in increase at the first sign of the shift and continues to do so throughout the inconclusive period. The metrics produced by the other algorithms will often increase but will fluctuate or decrease during this period. CUSUM also tends to be more robust to spikes in the pre-change signal as it requires a much larger, more sustained spike to trigger a false alarm than with the other algorithms. It is important to note however that there are some circumstances in which the CUSUM algorithm's performance is not desirable. Most notably, the algorithm cannot handle detecting multiple changes in the signal in rapid succession due to the fact that the sliding standard deviation window will still contain part of the previous change in the signal. The standard deviation will not have time to recover to pre-change levels and thus subsequent detections will be missed.

Table 2.3 highlights some of the advantages and disadvantages associated with each algorithm. Note that the "possible" entry shown for the power spectrum algorithm means that the feature indicated only occurs if the algorithm is monitoring a frequency band with a known signature occurring 
ahead of unstart, not just the low frequency band presented here. All told the CUSUM algorithm's robustness and timely detection make it an attractive option for change detection in the unstart problem. However due to its limitations detecting changes in flow conditions with frequent shifts it would perhaps be prudent to use one of the other algorithms to monitor the signal simultaneously. The CUSUM algorithm also offers little in the way of providing advanced detection of the approaching shock. Any algorithm or metric that could offer advanced detection would clearly be more desirable than those that do not.

Table 2.3: Summary of algorithm advantages and disadvantages

\begin{tabular}{|l|c|c|c|c|}
\hline & CUSUM & Power & S. Dev. & Mag. \\
\hline Universal threshold & $\mathrm{X}$ & & & \\
\hline Robust against spikes & $\mathrm{X}$ & possible & & \\
\hline Early detection & & possible & & \\
\hline Detect successive rises & & $\mathrm{X}$ & $\mathrm{X}$ & $\mathrm{X}$ \\
\hline Effective regardless of shape & $\mathrm{X}$ & & & \\
\hline Implementable online & $\mathrm{X}$ & $\mathrm{X}$ & $\mathrm{X}$ & $\mathrm{X}$ \\
\hline
\end{tabular}




\section{Chapter 3}

\section{Modeling Transient Dynamics with System Identification}

A mathematical model of shock motion would be a key tool in the prediction and closed-loop control of unstart. Such a model would ideally be derived from the full dynamics of flow within the engine, but the ability to accomplish such a derivation would be prohibitively difficult if not entirely intractable. Instead, a relatively simple model can be derived from the experimental data achieved from known inputs to the system in a process known as system identification. Here the input would be a selected transient aspect of the fueling process, and the output would be the time history of shock location (or metric indicative of this information). With time histories of both the input and output as well as a general model framework, the data can be used to guide the selection of the model parameters [27]. The goal is to select

a framework and parameters that consistently produce output values close the experimental output values for a given input. 


\subsection{Data Collection}

As a first step in this process, usable input and output signals must be produced and collected by the available hardware. Once obtained the data can then be used in the selection and fit a model. The various types of signals attempted and the procedures used to produce them with the hardware setups described are discussed in the following section.

\subsubsection{UT Hardware}

Several changes were made to the UT hardware to facilitate data collection for purposes of system identification. Previously the UT data used for change detection only experienced pressure changes due to the deflection of the flap. For identification the use of vortex generator jets (VGJs) installed along the side walls of the model was also included. Ultimately the VGJs are to be used as actuators in the attempt to control unstart, but for now they serve as an additional means of disturbance. A value of 1 has been assigned to the on condition for the VGJs and a value of 0 for off.

Only one type of input pattern has been attempted with the UT hardware: pulse trains. The pressure transducers collecting the output data are capable of operating at very high frequencies well beyond the needs of system identification. The input metrics (flap and VGJ operation) have not been recorded, but information about and time stamps of important events are available. These are used to recreate the input signals used in the identification algorithm. 


\section{Pulse Train}

Pulses of the shock's location were first attempted by deflecting the flap only. The VGJs were held constant in the fully "on" position throughout all such runs. In these runs the flap typically began at $0^{\circ}$, ramped up to a given angle, left at that angle briefly, and then ramped back down to $0^{\circ}$ three times. Time stamps of each ramp angle change were recorded and used to recreate the input signals. Figure 3.1 illustrates an example of the input and output data associated with a flap pulse train. Pulse trains were also attempted through
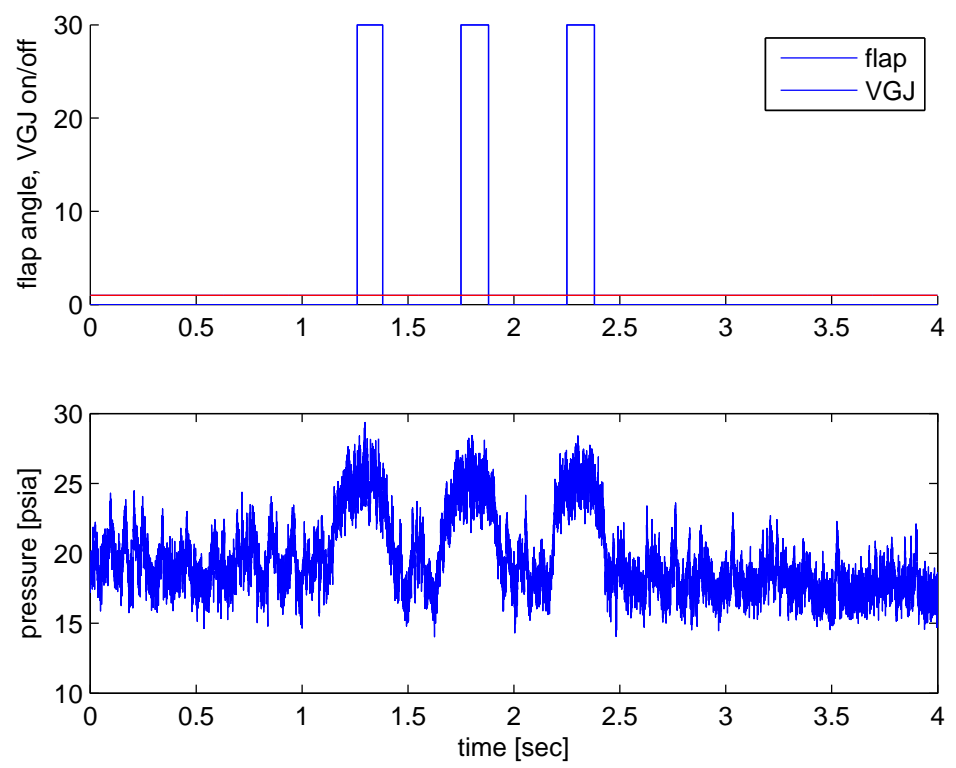

Figure 3.1: Pulse train created with flap movement

the use of the VGJs. Series of on/off cycles of the VGJs were completed with the flap typically held at a constant angle. The pulses were conducted a various frequencies ranging from $1 \mathrm{~Hz}$ to $100 \mathrm{~Hz}$. Figure 3.2 provides an example of a 
VGJ pulse train.
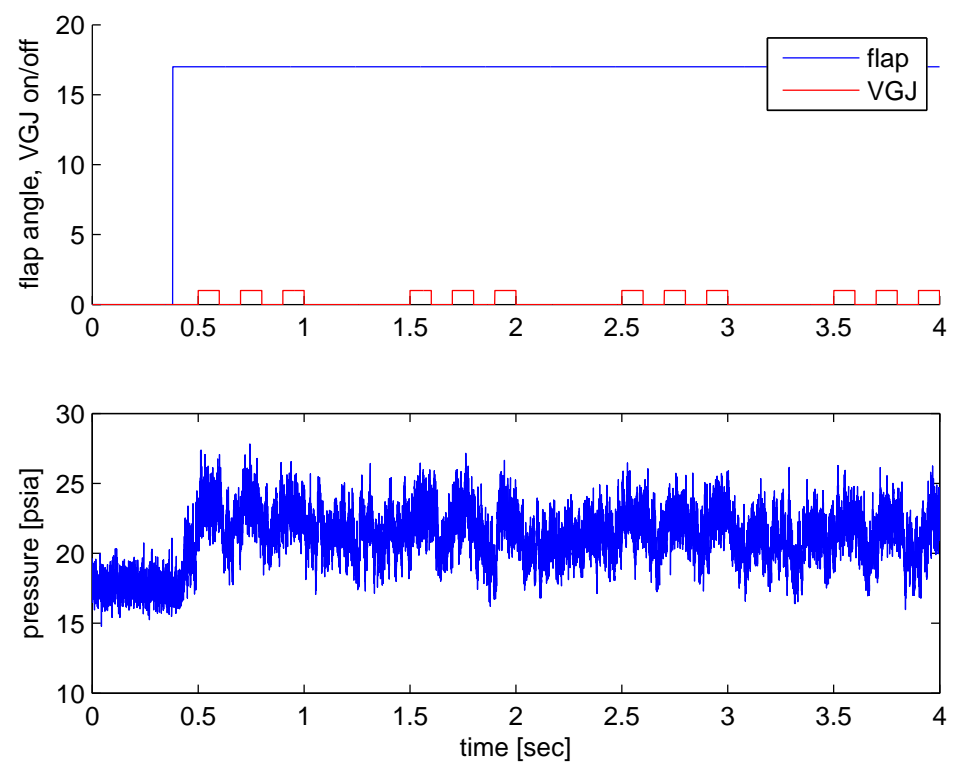

Figure 3.2: Pulse train created with VGJ cycles

\subsubsection{AFRL Hardware}

The AFRL hardware generated pressure changes for system identification by varying the fuel equivalence ratio. An air throttle is also used to create pressure changes but typically serves a disturbance similar to the VGJs in the UT case. Four types of inputs have been attempted with the hardware: a sinusoid, a step, an impulse, and a pulse train. The data acquisition system has a variable setting for the recording of the output metric and was typically run at approximately $2.5 \mathrm{kHz}$. Recording of the input metric however was initially limited to $1 \mathrm{~Hz}$. The system was later changed to permit $10 \mathrm{~Hz}$ input 
metric readings. Most (but not all) of the $1 \mathrm{~Hz}$ runs were repeated with $10 \mathrm{~Hz}$ collection, providing better resolution of sharp or fast changes. The hardware implementation and resulting signals are discussed here.

\section{Sinusoid}

Sinusoidal variations in fuel pressure were provided by the fuel-control system in an attempt to characterize the resulting response of the shock position. Attempts at sinusoidal inputs ranging from 0.1 to $0.4 \mathrm{~Hz}$ were completed as deemed feasible by previous bench tests. The $10 \mathrm{~Hz}$ input readings indicate that slightly higher frequencies might still provide recognizable sinusoids, but anything significantly faster would certainly be too skewed and damped. Figure 3.3 provides an example of the high-speed pressure history at a selected transducer as compared with the $10 \mathrm{~Hz}$ sinusoidal fueling input. Note that the initial pressure spike seen in this and subsequent figures is an artifact of starting combustion and is not a part of the desired fuel pattern. Figure 3.4 demonstrates how closely an actual sine wave can be used to describe a consistent portion of the input data. The sinusoidal shape of the output makes it possible to extract information about the magnitude and phase changes induced by the system. Ultimately a wider range of input frequencies would be necessary to provide enough data points to characterize these changes via frequency means such as Bode analysis. The current results do however confirm the feasibility of the frequency-based analysis methods and will be useful for system identification purposes. 

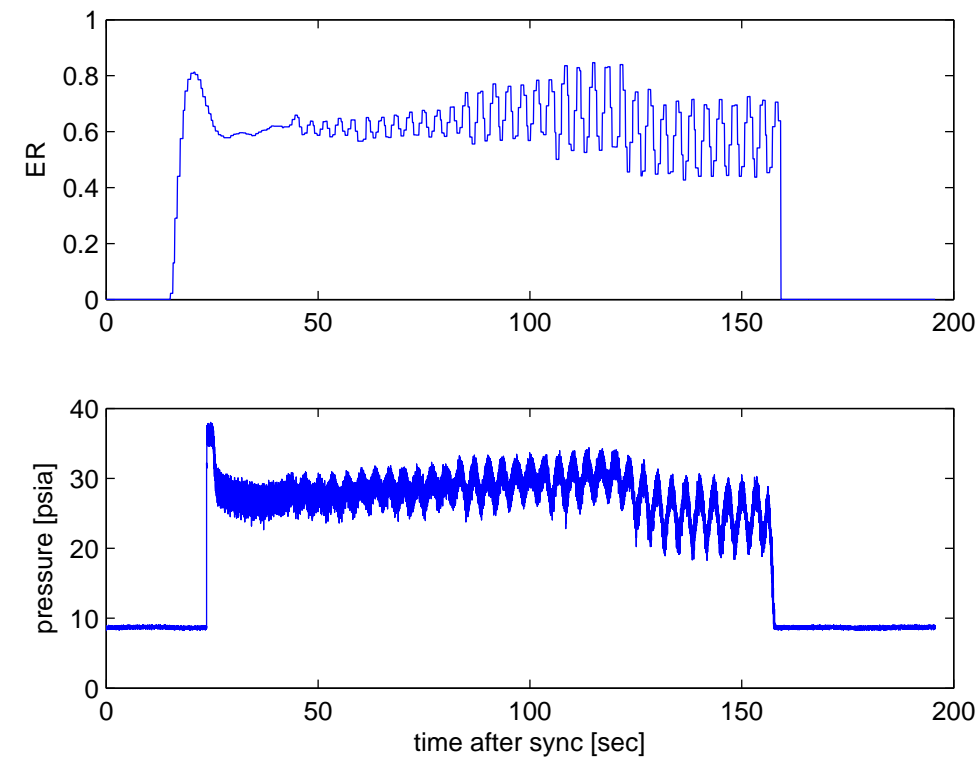

Figure 3.3: Example of sinusoidal fueling pattern, input: equivalence ratio, output: pressure at selected transducer

Step and Impulse

Two methods were employed to emulate step and impulse inputs to the shock location. The first method created a step-like input by allowing the shock position to stabilize at a given equivalence ratio then rapidly opening the fuel valve to a pre-specified position providing a slightly higher equivalence ratio. Using this method we bypass the slower fuel-control system which would have required a fuel set point change to achieve similar results. With the valve the "step" is completed over approximately 5-6 seconds, where a set point change would have taken approximately 10 seconds. Note that the magnitude of the step is not exact with the valve method, but this was much less critical than approximating the sharp jump of a step function. Although an improvement, 


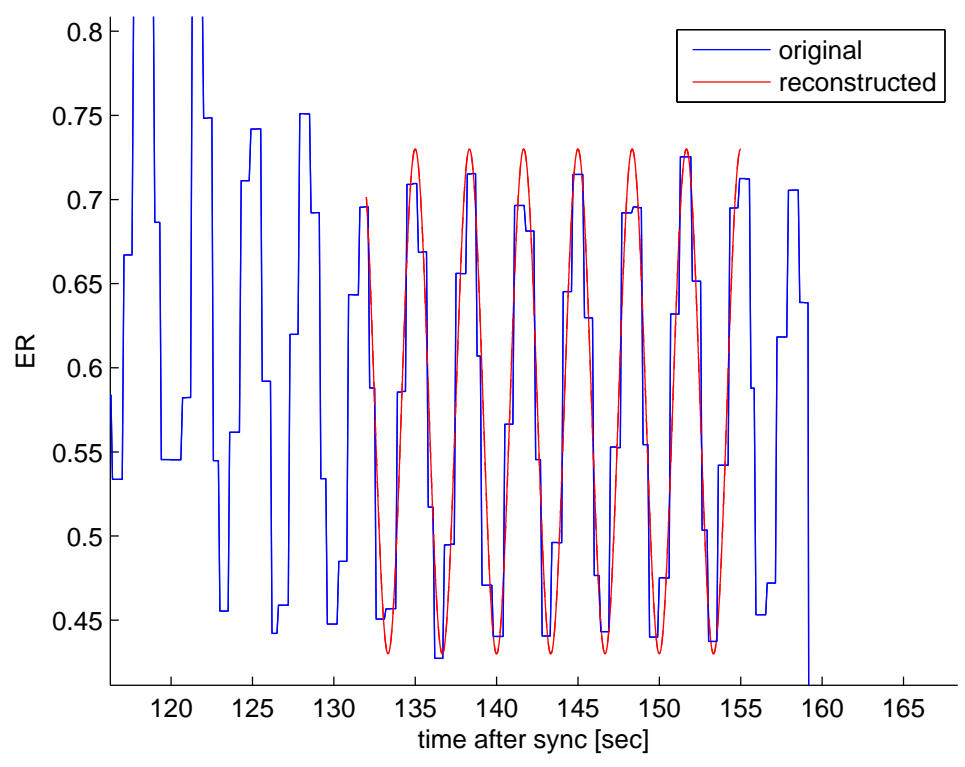

Figure 3.4: Sinusoid fit to sampled input data

the valve step still leaves much to be desired in approximating an actual step function. Impulses are clearly not possible with this method. Figure 3.5 illustrates the pressure history at one of the transducer locations passed during the direct valve step command. Formalizing the transient and steady-state characteristics of this step response will facilitate building a model for shock motion.

The second method created step and impulse-like inputs by opening the air throttle a pre-specified percentage and then rapidly closing it (during combustion). Again, it is much more critical for the changes to be sharp than for the magnitude to be exact. Indeed opening and closing the air throttle is much faster (approximately 0.5-1 seconds) than any similar pattern created 

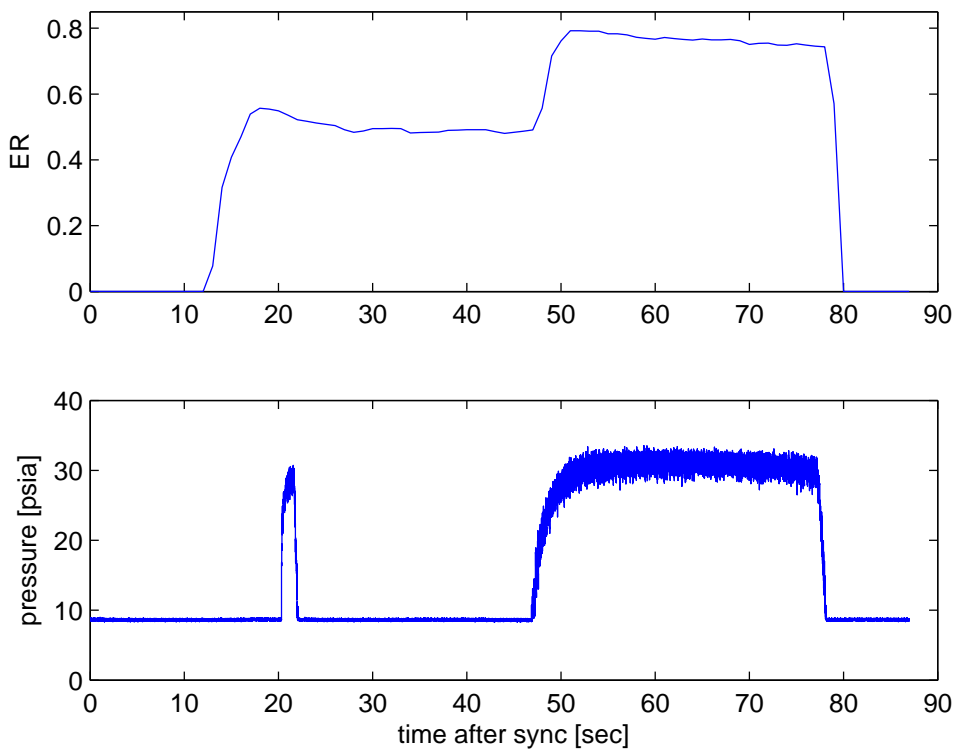

Figure 3.5: Step input created with direct valve manipulation

with fueling changes. However, the input information associated with the air throttle is the valve's mass flow instead of any type of pressure measurement. It is also important to note that the air throttle essentially acts as a disturbance to the system, and is ultimately not a controllable input during flight. Figure 3.6 illustrates several pulses of various magnitudes created in this manner though the pressure history at a selected transducer. Characteristics of these responses can be used similarly to that from the valve data as well as in a system identification framework to create a model for shock motion.

\section{Pulse Train}

The success of the air throttle approach (and $10 \mathrm{~Hz}$ change) makes possible other inputs that are based on the sharp changes seen in a step. Building 

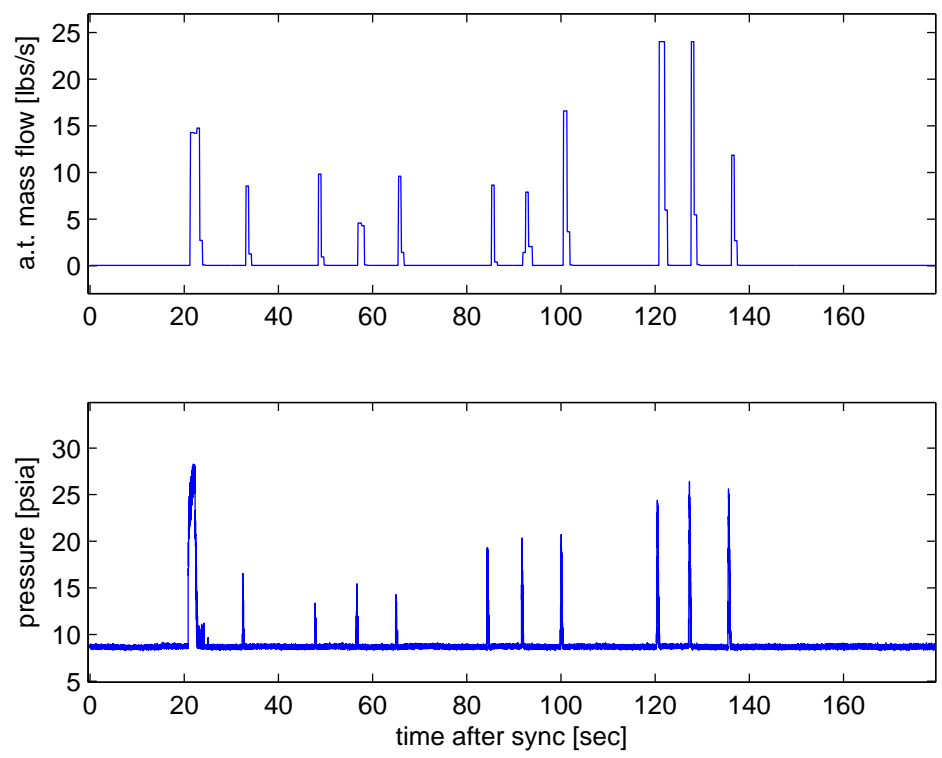

Figure 3.6: Pulses created with air throttle manipulation

on this shape a series of air throttle pulse trains was attempted where the air throttle was cycled open to a specified percentage for 5 seconds and then off for 5 seconds. The equivalence ratio was held approximately constant throughout the run so as to have the shock settle back to the same location between pulses. Several runs at various equivalence ratios were completed. Figure 3.7 shows an example of one such run by comparing the air throttle mass flow to the pressure history at a selected transducer. 

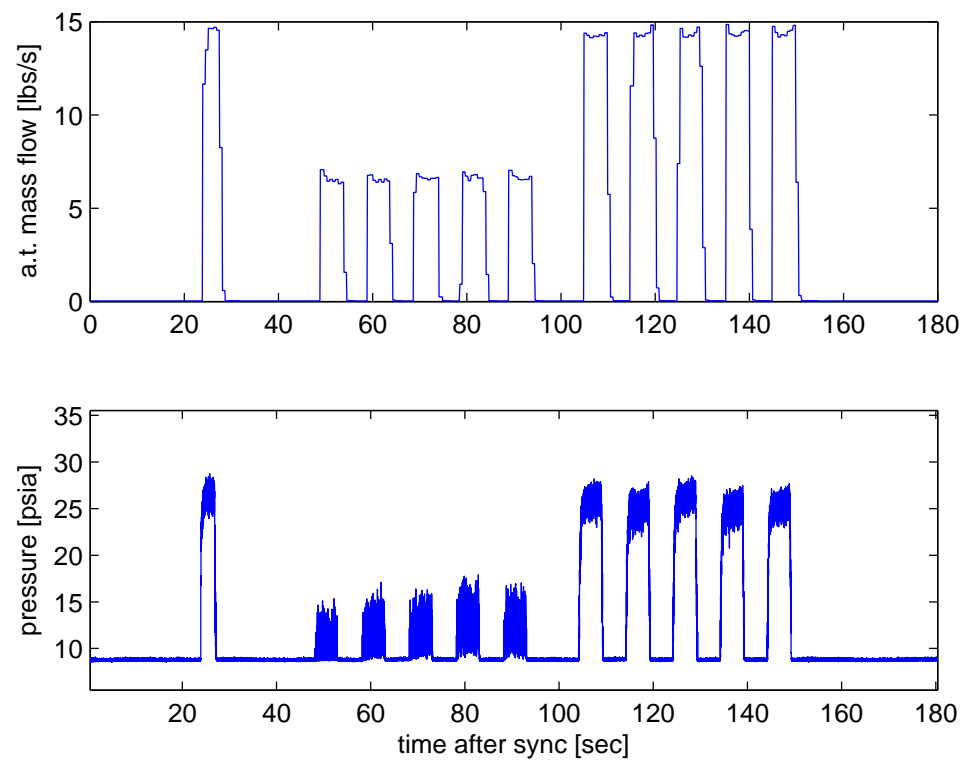

Figure 3.7: Pulse train created with air throttle manipulation

\subsection{System Identification}

\subsubsection{Overview}

In system identification, a system's observed input and output data are used to determine a mathematical model representative of the associated dynamic process. Many techniques and tools exist to "solve" for the parameters of the model, but most rely on statistical methods to choose model parameters that minimize the difference between the model's predicted output and known output in response to the known input. From this process it is clear that much can be gained from careful selection or design of system inputs. The general system identification in shown in Figure 3.8 [27]. All subsequent model fitting has been executed using MATLAB's system identification toolbox. 


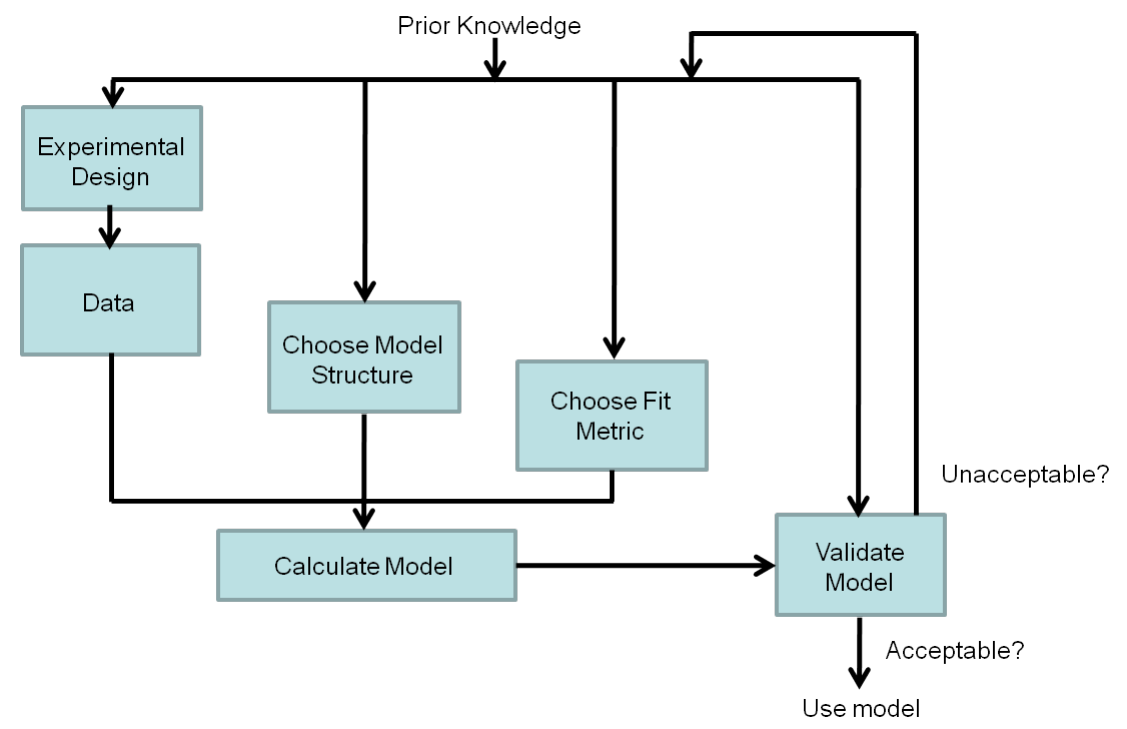

Figure 3.8: Flowchart of system identification process

In order to illustrate the system identification process, the following simple example will walk through the steps of identification and highlight modeling issues that will also be relevant to the unstart problem. For this purpose, consider a system described by the dynamics of the forced Duffing Oscillator, described in Equation (3.1). Here $u(t)$ is the input to the system and $x(t)$ is the output. The magnitude of the $\epsilon$ parameter determines how strongly nonlinear the behavior of the system will be. Note that $\epsilon=0$ leaves a linear dynamical system.

$$
\begin{gathered}
\ddot{x}(t)=-k x(t)-\epsilon x^{3}(t)+u(t) \\
u(t)=A \sin (\omega t)
\end{gathered}
$$

To simulate the identification process suppose that $u(t)$ and the corresponding $x(t)$ resulting from Duffing Oscillator are observed, but the rela- 
tionship between them is unknown. The goal is to estimate or identify this input-output relationship, which will hopefully be close to the actual relationship (known here to be Equation (3.1)). Following the steps for model identification illustrated in the flowchart, the first step is to design appropriate experiments to collect useful input-output data from the system. Here, this simply equates to selecting the parameter values of the input since the data $(u(t)$ and $x(t))$ is otherwise determined. Next, a viable model structure must be chosen. This example however will examine and compare several structures. Last, a metric to evaluate the quality of the model produced should be determined. The example will make use of MATLAB's built in performance rating for system identification where a fit percentage is computed from the model's predicted output compared the known output as described in Equation (3.2) [28]. The larger this percentage is, the better the model is.

$$
\%=100\left(\frac{1-\| y_{\text {model }}-y_{\text {actual }}||}{\left\|y_{\text {actual }}-\bar{y}_{\text {actual }}\right\|}\right)
$$

After the model has been generated, the flowchart states that the model should be validated on new data and then refined by repeating the identification process with improved input and structure selections if necessary. Here an extended period of input and output data will be generated in order to demonstrated the oscillator's dynamics. The first half of the data will be used to generate a model and the second half to validate the performance of the model.

To illustrate the importance of model structure selection, first consider the case of $\epsilon=0$. This assumption reduces the oscillator dynamics to a linear 
system as shown in Equation (3.3).

$$
\ddot{x}(t)=-k x(t)+u(t)
$$

If a linear model structure is chosen for identification purposes, as shown in Equation (3.4), it is expected that A, B, C, and D matrices will be found so that the model will produce an output nearly identical to the output data experimentally generated by the dynamics of the system.

$$
\begin{aligned}
\dot{\mathbf{X}}(t) & =A \mathbf{X}(t)+B u(t) \\
y(t) & =C \mathbf{X}(t)+D u(t)
\end{aligned}
$$

Now consider the nonlinear case where $\epsilon>0$ as seen in Equation 3.1. The size of $\epsilon$ will determine how closely the model behaves to the linear case. For a non-negligible $\epsilon$ it is expected that a linear model will not be sufficient and a nonlinear model structure will be necessary to achieve similar performance. However, a linear model may still be acceptable in this case if other concessions such as increased model order are made or if $\epsilon$ is relatively small.

Figure 3.9 shows the output data generated for 50 seconds of the prescribed input $u(t)$ in both the linear and nonlinear case. Data from 0-25 sec will be used to fit the models and data from 25-50 sec will be used to validate the models as mentioned previously. The validation result of a linear model structure fit to the linear case of the oscillator is illustrated in Figure 3.10. The left figure depicts the log of singular values of the data's covariance matrix. This is one way of characterizing how much of the input-output 


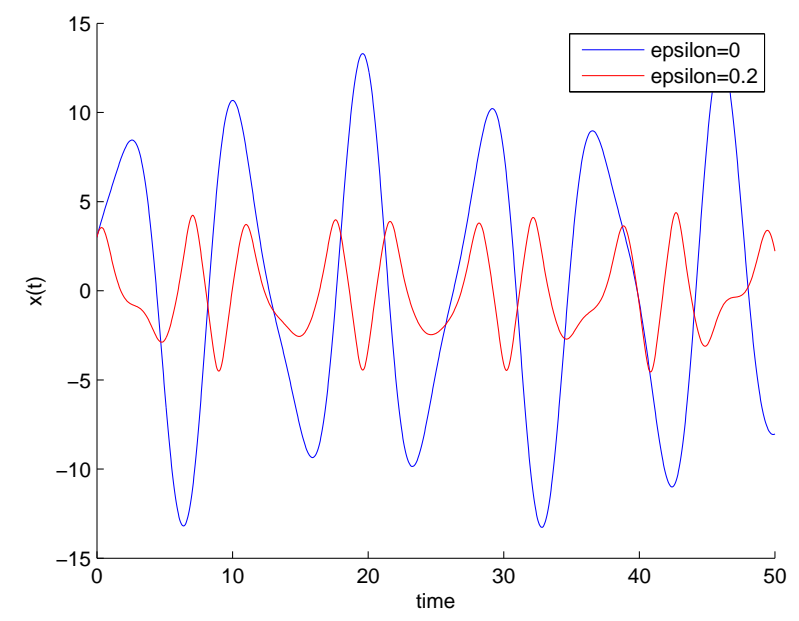

Figure 3.9: Output data generated for system identification example (0-25 sec: generation, 25-50 sec: validation)

behavior is captured by a given number of states, and is used to determine the model order selected. The default selection of order 2, shown in red, has been used here. As expected, the output of the resulting model matches the actual output very well. Further, the dimensionality of the identified linear model exactly matches that of the actual dynamics. The result of the linear model structure applied to the nonlinear case of the oscillator in shown in Figure 3.11. Here the suggested model order selection of order 5 is used. The fit rating of $61 \%$ indicates that the linear model arrangement is much less successful and other model structures should be investigated. Finally, Figure 3.12 illustrates the more successful fit of a nonlinear model structure to the nonlinear oscillator case. The fit rating of $75 \%$ indicates that performance of the nonlinear structure is relatively improved compared to the linear one. There are many existing nonlinear structures available, and selection should 
be at least partially motivated by any known information about the dynamics of the system in question. Here a nonlinear ARX model structure with a wavelet network nonlinearity estimator has been used though details about this particular structure and motivation for its selection are omitted. Model selection for the unstart problem will be discussed in detail in the next section.

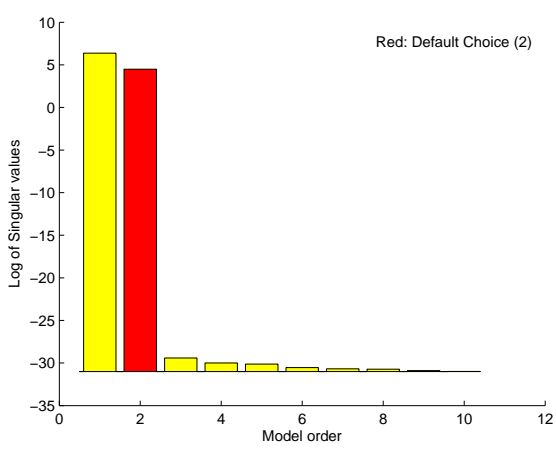

(a) Model order selection

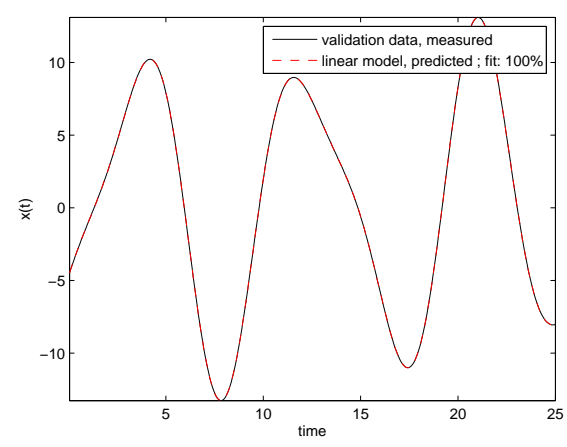

(b) Model output vs. data

Figure 3.10: $\epsilon=0$, linear model structure

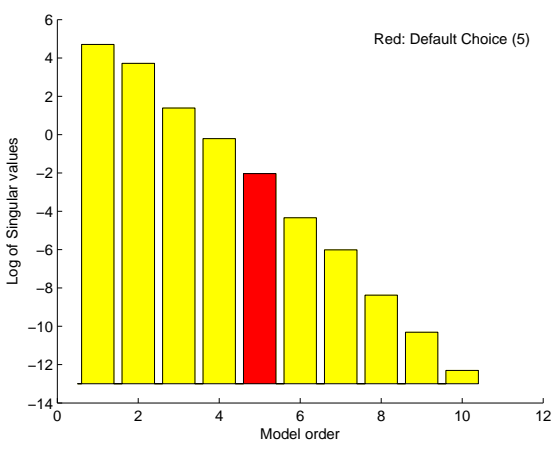

(a) Model order selection

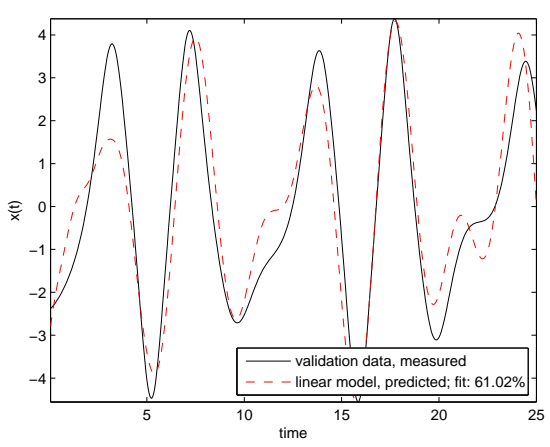

(b) Model output vs. data

Figure 3.11: $\epsilon=0.2$, linear model structure 


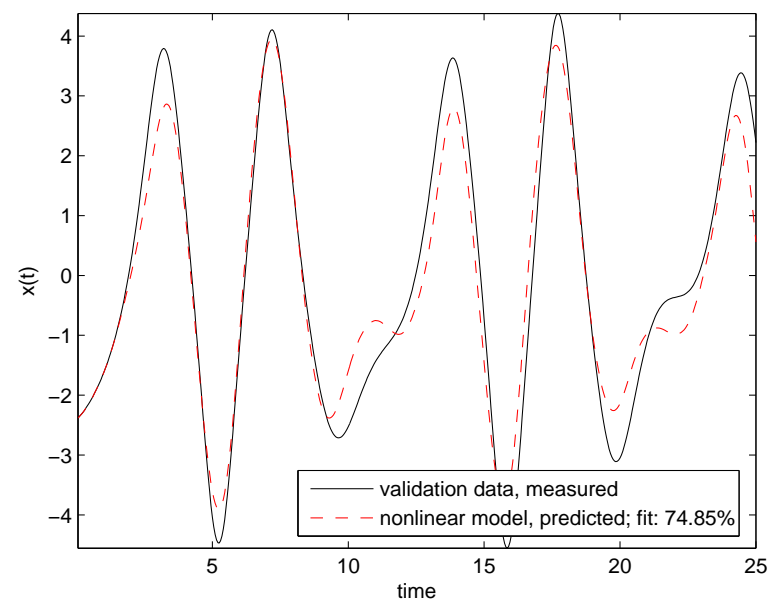

Figure 3.12: $\epsilon=0.2$, nonlinear model structure

\subsubsection{Model Selection}

The system identification process can be easily adapted to the unstart problem. Figure 3.13 revisits the system identification flow chart presented earlier and reformulates each of the blocks in terms of the problem at hand. Note that each of the steps in the process outlined is well defined, except for the input provided and model structure to be used. In this section, aspects of the unstart problem affecting the selection of input and model structure are discussed and a successful setup is identified. Ultimately, the goal is to find a single model structure that permits adequate prediction of transducer output when applied to an input of any pattern. Ideally the model structure would be suitable for all of the transducer locations and across various hardware setups. The first step however, is to begin with the most basic structure: a linear model applied to a single transducer's data on a single run of one 


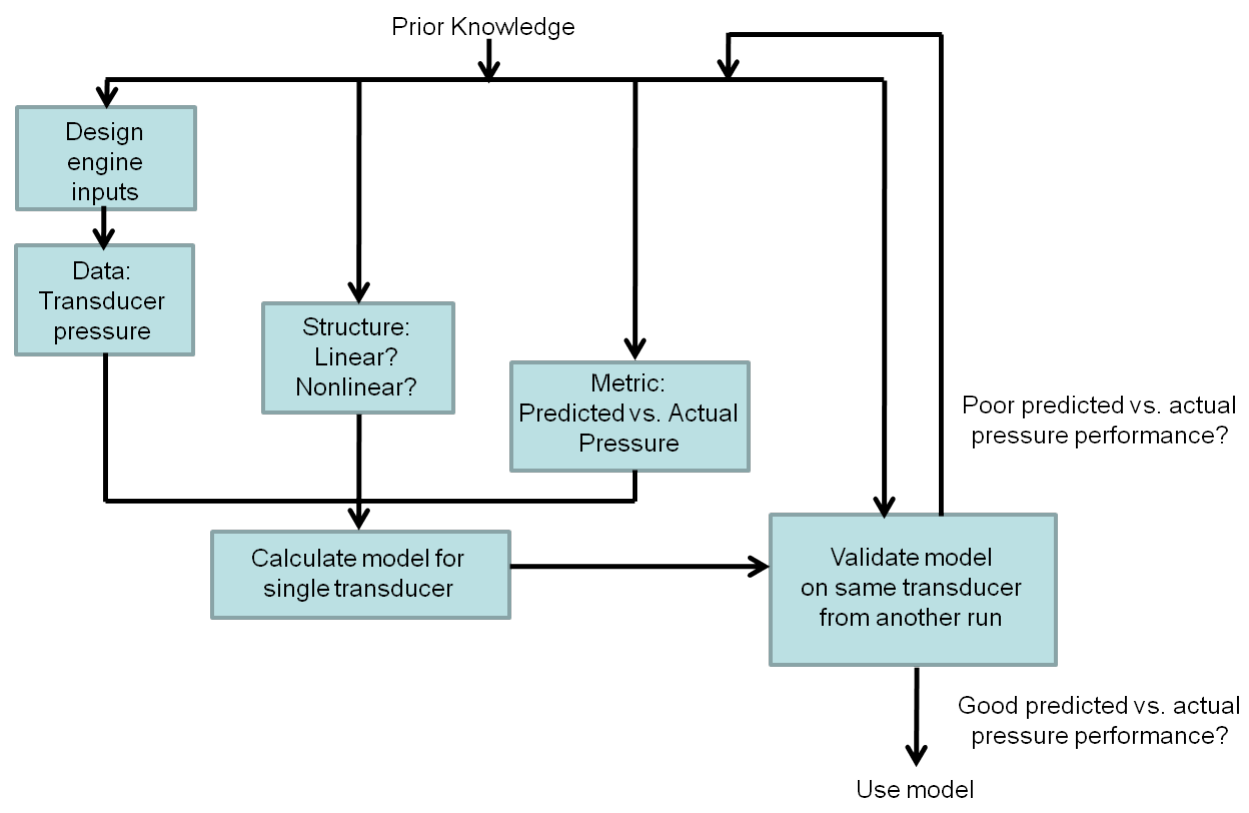

Figure 3.13: Flowchart of unstart specific system identification

one input pattern. Should this setup prove to be successful (in terms of the predicted output matching the experimental output) in the standalone case, then the linear structure's success should be further assessed (for example on data from multiple runs) until the ideal case has been satisfied. Should the linear model structure fail, the features that caused the failure should attempt to be identified and remedied through information provided as input or by selecting a different model structure. The process begins again and continues until a successful setup is hopefully found. All model fitting has been completed with MATLAB's system identification toolbox.

Attempts to use individual data runs collected to produce a model with acceptable predicted output under the basic setup conditions have been largely 
successful. Typically, the linear structure can be used to predict the output of a single transducer without issue when varying a single fueling parameter such as equivalence ratio or fuel valve pressure and allowing it to also be available as an input. However, performance of the standalone models quickly diminishes as it is applied to a wider range of transducer locations or input patterns. The resulting behavior of the predicted output in these cases makes it clear that the linear model is at fault for the failure to capture all of the output's features. For example, Figure 3.14(a) depicts such a case where both a linear (3rd order) and nonlinear (nonlinear ARX model with 11-unit sigmoid network estimator) model have been fit to the data from one transducer in a sinusoidal fueling run. In this particular case, the shock is beyond the selected transducer for the duration of the data selected. Both models easily accommodate this situation and thus use of a nonlinear model would certainly be unnecessary. However, Figure 3.14(b) shows the results from fitting the same model structures to another transducer from the same run. Here the shock passes back and forth over the transducer over the course of the selection. Because of the zeroed portions of the output that correspond to the shock retreating behind the transducer it is expected that a linear model will not be able to accurately represent the output. The figure illustrates that the indeed only the nonlinear model successfully captures the flat line behavior. The same would also be the case for a transducer the shock never passes. Therefore, for this reason alone a linear model is not sufficient and nonlinear model structures must be explored instead. 


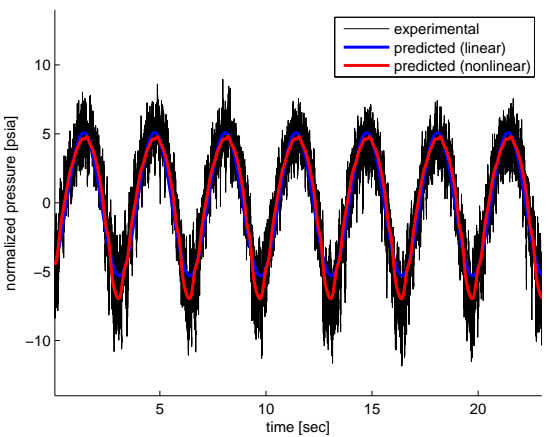

(a) Downstream transducer

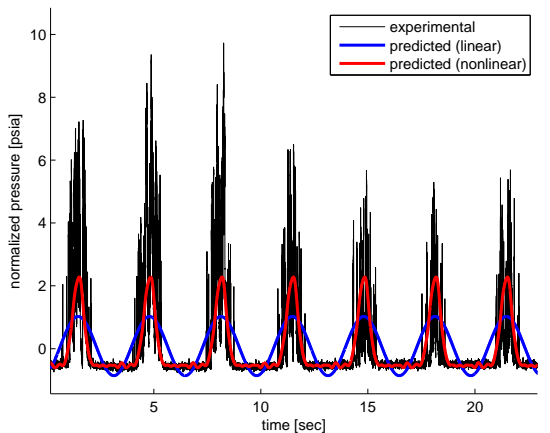

(b) Upstream transducer

Figure 3.14: Illustration of transducer location effect

Although standalone models do work in the specific instance they were created for, a model that could be applied to multiple transducers or multiple input patterns would be much more useful. Generation of models that could accommodate this range would be more feasible if multiple runs could be incorporated into the calculation of a single model. However, there are two main obstacles that stand in the way of combining data from different runs. First, only runs that have one varied input parameter and can currently be used to produce a single model for a specific transducer. For example, two runs that both have the same air throttle steps executed in fueling conditions with the same equivalence ratio can be used together. The same two runs could not be used together if the equivalence ratio of one was higher than the other. Second, for reasons illustrated by Figure 3.14, one single-output model cannot take the same input and predict the differing outputs of two separate transducers. Each transducer must currently have its own model. 
Various methods to address these issues have been explored. Possible solutions for the two-parameter problem include modeling additional parameters as additional inputs and model interpolation across similar standalone models. Possible solutions for the transducer problem include incorporating experimentally established relationships between shock location and various fueling parameters into the modeling process. After experimenting with each of these solution methods, only the two-parameter multiple input change reliably improved the predicted output matching of models generated and applied to more than one run. Further experimentation with various model structures (now taking more than one input and using more than one run to generate a model) quickly revealed that one particular type of nonlinear model structure, namely the Hammerstein-Wiener dynamic model, most reliably produced identification that successfully predicted outputs across multiple runs. Discussion of the Hammerstein-Wiener model and examples of the results achieved with this particular model structure are discussed in the subsequent sections.

\subsubsection{Hammerstein-Wiener Model}

The Hammerstein-Wiener model creates a nonlinear model from a linear base structure by permitting nonlinear accommodations on both the input and output signals. The model is thus a combination of the Hammerstein structure which has an input nonlinearity and then a linear block, and the Wiener structure which has a linear block and then an output nonlinearity. The block diagram in Figure 3.15 outlines the Hammerstein-Wiener structure 
[29], [30].

\begin{tabular}{|c|c|c|c|c|c|}
\hline$u(t)$ & Input & $w(t)$ & Linear & $x(t)$ & Output \\
\hline & $\mathrm{g}(\mathrm{u})$ & & $\mathrm{P}(\mathrm{q})$ & & $f(x)$ \\
\hline
\end{tabular}

Figure 3.15: Block diagram of Hammerstein-Wiener model structure

The result of the input block is a static nonlinear function, $w(t)=$ $g(u(t))$, that is defined separately for each of the $n_{u}$ number of input signals. The choice of nonlinear function is dictated by the problem and will be discussed later in the context of specific data. The linear block is a transfer function, $P(q)$, where there is a separate transfer function defined for each combination of one of the $n_{u}$ inputs and one of the $n_{y}$ outputs. Each transfer function is of the form

$$
P(q)=\frac{\beta(q)}{\alpha(q)}=\frac{\beta_{1} q^{-1}+\beta_{2} q^{-2}+\ldots+\beta_{n} q^{-n}}{1-\alpha_{1} q^{-1}-\alpha_{2} q^{-2}-\ldots-\alpha_{n} q^{-n}}
$$

where $n$ is the order of the linear block and $q$ is the time shift operator. In order for the linear block output $x(t)$ and model output $y(t)$ to be of the same dimension, a separate $x(t)$ is defined for each outputs according to Equation (3.6). Note that this relationship also allows for the inclusion of a delay, $n_{k}$, for each input [30].

$$
x(t)=\sum_{i=1}^{n_{u}} \frac{\beta_{i}(q)}{\alpha_{i}(q)} w_{i}\left(t-n_{k_{i}}\right)
$$

The output block is again a static nonlinear function of the form $y(t)=f(x(t))$ which will also be selected based on the specific application. The function is defined separately for each output signal [31]. 


\subsection{Results}

\subsubsection{UT Model}

As an example of the two-parameter solution success, consider the same transducer from three separate pulse train runs. Input information and the pressure histories at the selected transducer from two of the runs are combined and used to generate a model with two inputs (flap and VGJ motion) and one output (pressure at selected transducer). In the first run the pulses are produces by the flap, in the second by the VGJs, and in the third by a combination of the flap and the VGJs. The combination run is not used in model generation, but is instead used for blind validation of the model produced. Here the data is given a Hammerstein-Wiener model framework to fit with a piecewise linear input nonlinearity estimator, a fifth order transfer function with unit input delay, and a piecewise linear output nonlinearity estimator. Selection of the input nonlinearity form was apparent from the structure of the input signals used. Selection of the output nonlinearity form was determined largely from trial and error. However, any form that predicted substantial erroneous pressure spikes between pulses was immediately discarded. Forms that provided desirable features such as trend matching or minimal delay between predicted and actual pressure rises were pursued further. The model structure was adjusted until acceptable predicted output was achieved for both of the model generation runs.

Figure 3.16 shows the inputs, resulting output, and predicted output of the first run used in model generation. Figure 3.17 shows the same for the 
second model generation run. In both cases the predicted output only exhibits the pulse shapes causes by the inputs, as desired. Note that prediction of the pressure changes is slightly delayed. Any delay in prediction could be detrimental to the unstart problem and further investigation of the reason for this delay and its elimination are necessary. The magnitude of the pressure changes is also not well represented by the model's output, but this type of agreement is of much less concern than agreement of timing of pressure changes. Figure 3.18 shows the results of the same model blindly applied to the third set of data as a means of validation. The model successfully predicts the pressure changes, though similar delay and magnitude issues are noted. In particular the magnitude of the predicted output during the VGJ pulses is not well represented. This is likely due to the fact that the flap is deflected during these pulses, but neither of the runs used to produce the model contained a similar feature. Adding an additional generation run with simultaneous flap and VGJ motion would likely fix the problem. The specific model structure and parameter values are given in Appendix A. 


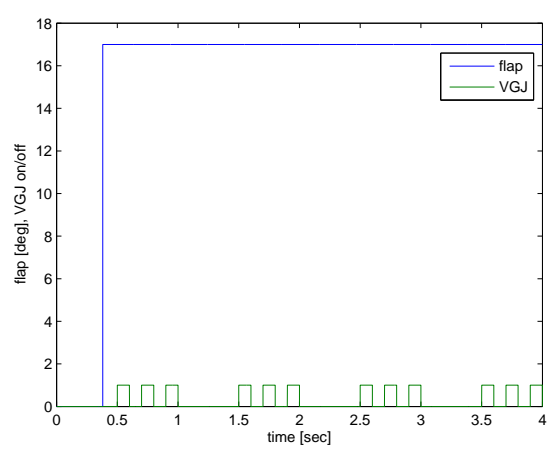

(a) Input

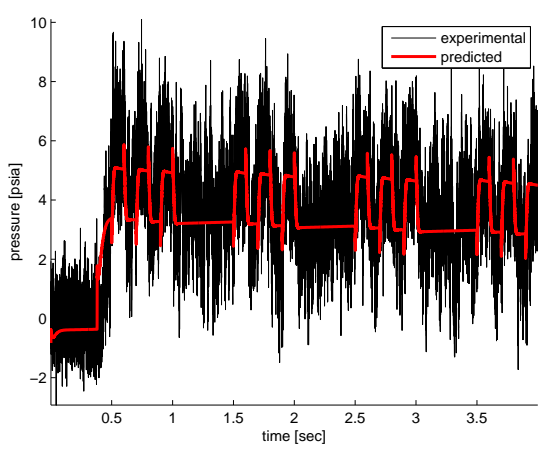

(b) Output

Figure 3.16: Model generation run, flap constant with VGJ pulses

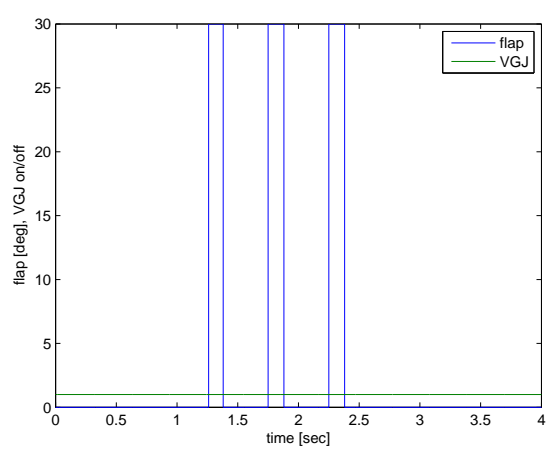

(a) Input

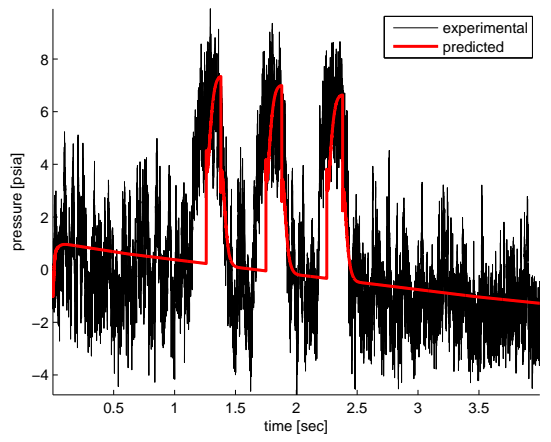

(b) Output

Figure 3.17: Model generation run, flap pulses with VGJs constant 


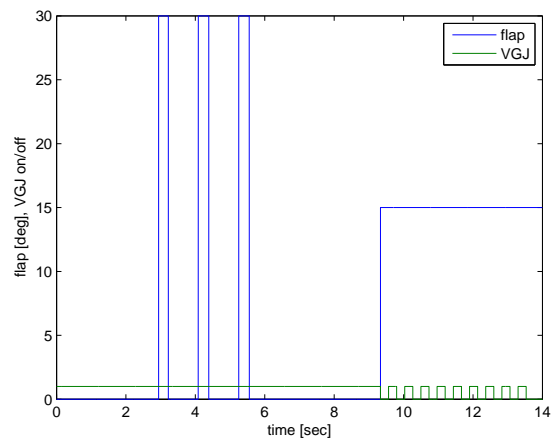

(a) Input

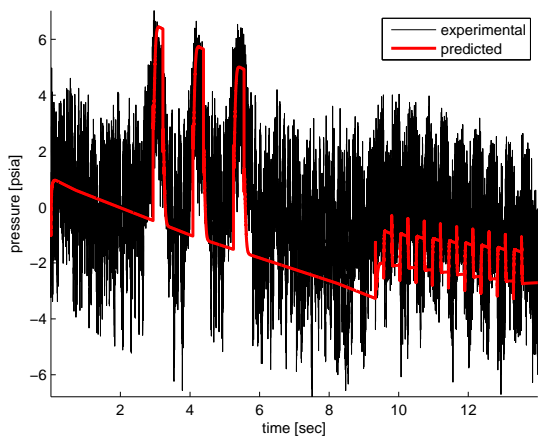

(b) Output

Figure 3.18: Model validation run, variation in both flap and VGJs

\subsubsection{AFRL Model}

As a second example of the two-parameter solution success, consider the same transducer from three separate air throttle pulse train runs. Each was completed at a different equivalence ratio and with a slightly different pulse pattern. Here a similar model is determined from the first two runs using two inputs (air throttle mass flow, equivalence ratio) and one output (pressure at selected transducer). Again the third run is not used in model generation, but is instead used for blind validation of the model produced. The two combined runs are given a Hammerstein-Wiener model framework to fit with a sigmoid network input nonlinearity estimator, a third order transfer function, and a dead zone output nonlinearity estimator. Selection of the output nonlinearity form here was clear due to the all-or-nothing behavior of the pressure characteristic of the AFRL data that depends on the shock being upstream or downstream of the transducer. The input form from trial and 
error with similar guidelines as discussed in the UT case.

Figure 3.19 shows the inputs and resulting output and model prediction of one of the two model generation runs. Figure 3.20 shows the same information for the other model generation run. The particular transducer used here was specifically chosen as the equivalence ratio used for the first run allows the smaller pulses to produce movement in the selected transducer's signal where the equivalence ratio used in the second run does not. This illustrates that the particular model produced can appropriately accommodate flat line behavior. Finally, Figure 3.21 shows the inputs, output, and prediction results of the same model blindly applied to the third run that was not used in generation. In this case the equivalence ratio ensures that the shock is always in front of the selected transducer. The model correctly predicts no flat line behavior even though this was not the case in the model generation runs. The model also captures the abrupt rise and fall of the pressure signal associated with the pulses but fails to accurately predict the magnitude of the signal after the jumps. As discussed, this failure is not of great concern as the abrupt changes are ultimately the feature used to identify shock motion and eventually unstart. The specific model structure and parameter values are given in Appendix B. 


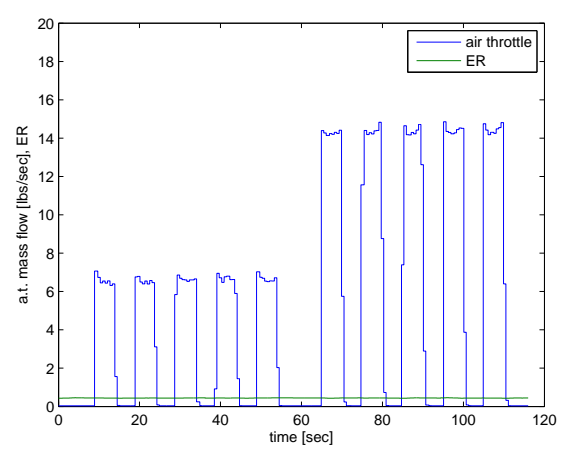

(a) Input

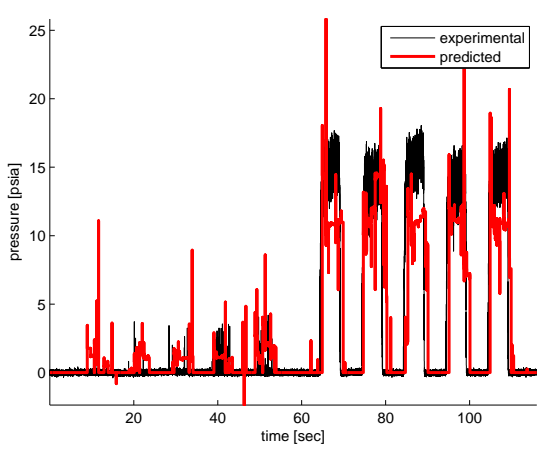

(b) Output

Figure 3.19: First model generation run

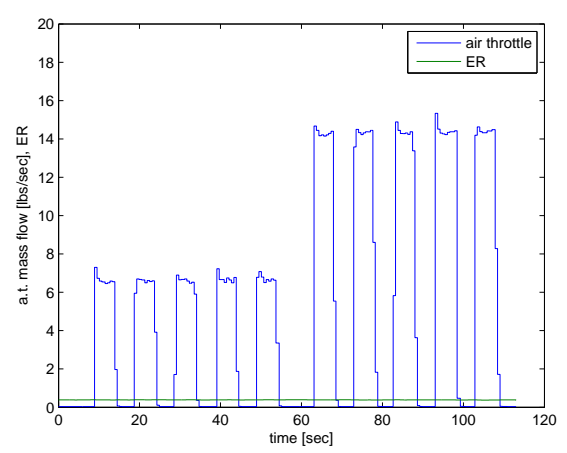

(a) Input

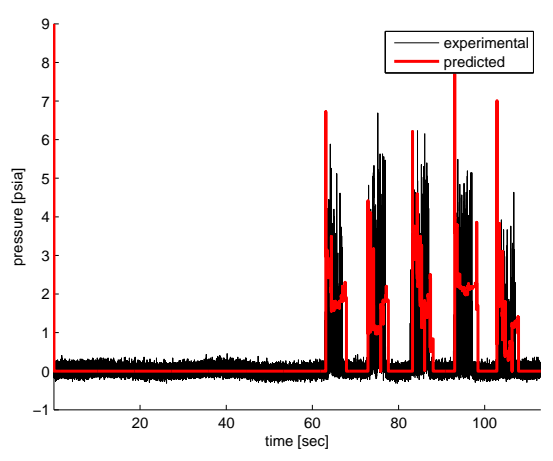

(b) Output

Figure 3.20: Second model generation run 


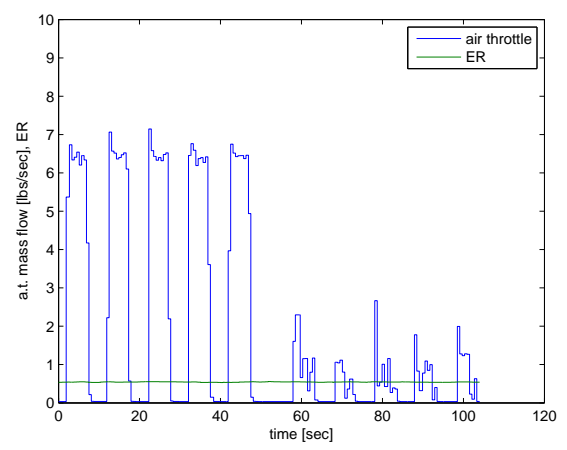

(a) Input

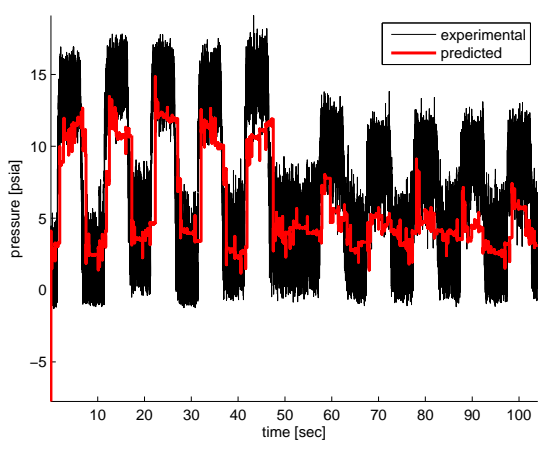

(b) Output

Figure 3.21: Model validation run 


\section{Chapter 4}

\section{Modeling Transient Dynamics with Structured Nonlinearities}

\subsection{Motivation}

The Hammerstein-Wiener model structure and largely automated model generation is certainly a useful method for modeling unstart. However, little information is gained concerning the system's dynamics since all of the work to characterize the input-output relationship is taken care of by the system identification process once the structure is selected. The ability to instead create and fit a model based on particular insights into the system's nonlinear behavior could potentially allow for improved representation of the system's dynamics. As an alternative to the models developed fully from system identification, this chapter presents some preliminary attempts to create simpler, more transparent models by analytically determining as much of the inputoutput relationship as possible.

\subsection{Structure Simplification}

The ability to simplify the model structure by analytically describing some nonlinear aspects of shock motion is made possible by a key observation 
of the shock's behavior: the shock behaves linearly under certain conditions. This observation was briefly mentioned in the previous chapter and was illustrated in Figure 3.14. If the conditions that cause the linear model to fail could be identified and mathematically described then there is the potential that the input and output nonlinearities of the Hammerstein-Wiener system identification model could be replaced by these functions. This would leave only the linear portion of the model to be found through system identification - a much simpler and well understood task.

\subsubsection{Identify Nonlinear Effects}

The feasibility of the simplified structure relies on the ability to determine what factors contribute to the nonlinear effects seen in unstart data. The extent to which these factors cover all sources of nonlinearity will impact the simplified model's chance of success. Most notably, if a significantly contributing factor fails to be included then the model's efficacy will be limited. However, identification of a nonlinear factor is of little use if its effects cannot be separated from other factors or adequately described using the resources available.

After working with the data sets used for system identification, the following two factors have been identified as the primary impediments to the success of the linear model at all times. Other factors have been identified but either cause a much smaller impact or are difficult to independently discern in the data. 
1. Shock location behind transducer

When the leading edge of the shock is behind the pressure transducer location being modeled, the pressure output is essentially unaffected by inputs or disturbances that do not push the shock in front of the transducer. Only the portions of these signal that occur when they have caused large enough change in shock location to advance it up to the transducer will be represented in the output. As long as the shock is ahead of the transducer the output behaves in a linear fashion. However the output will respond as if there are no changes to the system when the shock is behind the transducer. Although this behavior is described in an abrupt on/off manner there is a small amount of uncertainty as to when the output is affected by shock location. In general it appears that a shock location just behind the transducer will still permit inputs and disturbances to influence the output albeit in a reduced manner. However the precise shock location is never exactly known in most of the data runs available. It is typically approximated based on pressure readings at locations where transducers are present and experimentally known patterns based on fueling and flow conditions. The perceived slightly preceding pressure change could simply be due to the inability to resolve the shock location accurately.

2. Effect of inputs or disturbances varies based on location or conditions The impact of some inputs and disturbances can vary at different locations throughout the inlet or at different fueling and flow conditions. The 
air throttle in particular is sensitive to these parameters. Assuming that the shock is in front of the transducer (either prior to or because of air throttle use) the magnitude of the output change due to the air throttle can vary based on the previously mentioned factors. This variation does not restrict the use of a linear model per say though it does mean that the a slightly different linear model would be needed to achieve the same level of output matching whenever any of the factors change. An output scaling relationship based on the factors influencing air throttle impact is necessary if the same linear model is to be used at all times.

\subsubsection{Simplified Model Structure}

The Hammerstein-Wiener model structure employed previously made use of nonlinear function on both the input and the output of the linear block. However each of the nonlinear factors just identified can each be modeled as functions that modulate the output. The nonlinear function on the input of the linear block is therefore not necessary and is dropped from the model structure being considered here. The result is shown in Figure 4.1. This type of structure is known as a Wiener model.

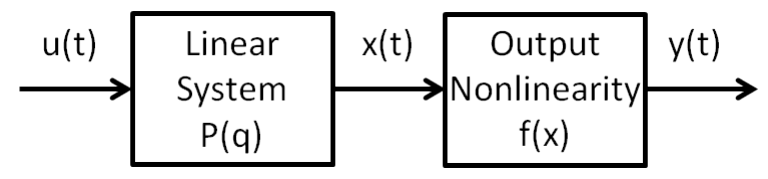

Figure 4.1: Simplified model block structure

As the linear portion of the model will still be obtained through system 
identification all that is left is to express the nonlinear factors as a single function that operates on the output signal of the linear block. To simplify terminology, the discussion of this process will be done in terms of the AFRL hardware with equivalence ratio and air throttle inputs. However the same discussion readily applies to the UT hardware by replacing these inputs with flap motion and VGJ actuation, respectively.

To convert the first nonlinear factor into an expression, consider each of the ways in which the shock can be advanced in front of the transducer location, $X_{t}$, in question. Either the equivalence ratio, $\phi$, can sufficiently move the shock by itself or the air throttle, $a$, can act in addition to the equivalence ratio to accomplish the motion. Note that the air throttle would never be operating without fueling once the flow has been started. This leads to separation of the output nonlinearity function into three cases: shock location due to equivalence ratio, $X_{s}$, ahead of transducer, shock location due to equivalence ratio behind transducer and air throttle active, and shock location due to equivalence ratio behind transducer without air throttle activity. When the equivalence ratio-based shock location is already ahead of the transducer the pressure output can simply be calculated from the linear model as neither of the nonlinearities are encountered. The case where the shock is behind the transducer and the air throttle is active however must be further subdivided. Here the magnitude of the air throttle will cause the shock location to move further upstream by an additional distance denoted here as $X_{\Delta}$. The calculation of this additional distance is where the second nonlinear factor must be 
considered. The size of the distance is not only dependent on the magnitude of the air throttle, it is also dependent on the shock's current location in the inlet. This secondary dependency can be indirectly included in the calculation of $X_{\Delta}$ through dependency on equivalence ratio. If the additional distance is enough to push the shock past the transducer then the output will display some of the effects of the inputs, though not the full effects as calculated by the linear model. Specifically, the output will reflect only downscaled portions of air throttle input which can be described in terms of $X_{s}, X_{t}$, and the air throttle's magnitude (again making use of the condition-dependent response described by the second nonlinear factor). If the addition distance is not enough to move the shock past the transducer then no response will bee seen in the output. Finally, if the equivalence ratio-based shock location is behind the transducer and there is no air throttle activity to move it further then again the output will display no response to the inputs. The cases described here are summarized in the final nonlinear function applied to the output of the linear block shown in Equation (4.1) for a given sample $k$.

$$
y_{k}= \begin{cases}\text { if } X_{s, k}<X_{t}: \\ x_{k} \\ \text { if } X_{t}<X_{s, k} \text { and } a_{k} \text { exists: } \\ f_{1}\left(X_{s, k}, X_{t}, a_{k}\right) & \text { if } \left.\left(X_{s, k}\right)-X_{\Delta, k}\right)<X_{t} \\ 0 & \text { if } X_{t}<\left(X_{s, k}-X_{\Delta, k}\right) \\ \text { if } X_{t}<X_{s, k} \text { and no } a_{k} \text { exists: } & \\ 0 & \end{cases}
$$

Remember that during unstart the shock moves upstream (towards $x=0$ ). So, to be behind a transducer is to have a larger $x$ location and to be in front is 
to have a smaller $x$ location. Also note that certain parameters have additional dependencies:

$$
\begin{aligned}
X_{s, k} & =f_{2}\left(\phi_{k}\right) \\
X_{\Delta, k} & =f_{3}\left(a_{k}, \phi_{k}\right) .
\end{aligned}
$$

\subsection{Application to AFRL Data}

The simplified model will only be shown as applied to the AFRL data set used in system identification. Significant amounts of data are necessary to help define the relationships used in the output nonlinearity of the simplified structure, and sufficient data is not yet available for the UT data set.

\subsubsection{Obtaining Linear and Nonlinear Elements}

The linear portion of the model is formed via system identification with a linear model structure. This is accomplished with MATLAB's pem function which relies on an iterative prediction-error minimization scheme to determine model parameters. As with the nonlinear system identification model structure previously used, two data runs are used to fit the model. It is important that runs in which neither of the nonlinear factors are encountered are used. The model order is the only additional parameter that must be specified.

The nonlinear function as described in Equation (4.1) requires that three yet unknown expressions be developed in terms of known signals: $X_{s}$, $X_{\Delta}$, and the output scaling in the case that the air throttle advances the shock 
ahead of the transducer. This can be accomplished with regression analysis as long as a sufficient amount of data is available.

Equivalence ratio-based shock location $X_{s}$

For $X_{s}$ the shock location due only to the equivalence ratio input signal is desired. The relationship between the two is determined by identifying the equivalence ratio when the shock passes a given transducer over many runs. The shock is said to be at the location indicated by a specific transducer at the instant an abrupt rise occurs in that transducer's pressure reading. The equivalence ratio at that instant can be pulled from the input signal. Regression analysis is used to determine a function that adequately relates the independent variable, equivalence ratio, to the dependent variable, shock location. There are clearly other factors that influence shock location. The method of determining shock location described here is also admittedly crude. However even the imprecise relationship determined by this analysis will be shown to be sufficient for modeling. Figure 4.2 illustrates some of the data collected and a quadratic polynomial that well describes the trend. The quadratic polynomial is then used in the $f_{2}$ expression in the nonlinear output function to determine the projected shock location for a given equivalence ratio. Details of the polynomial fit are given in Appendix C.

\section{Air throttle-induced additional shock motion $X_{\Delta}$}

For $X_{\Delta}$, an expression for the distance the shock moves beyond the shock location due to the equivalence ratio is needed. This can be adequately 


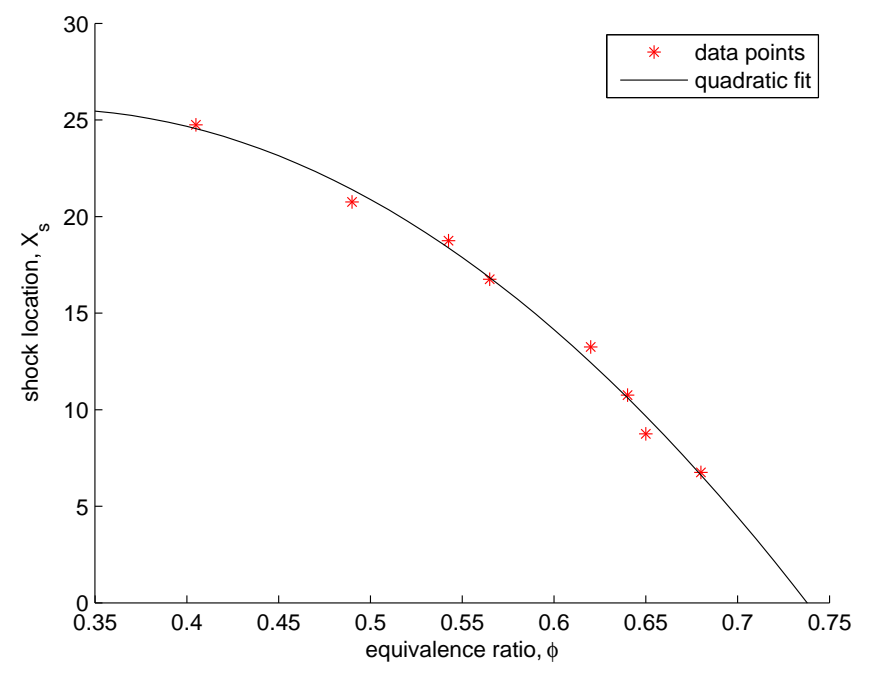

Figure 4.2: Quadratic fit describing equivalence ratio vs. shock location relationship, $R^{2}=0.992$

described (for this data set) in terms of the air throttle mass flow and equivalence ratio input signals. The data points used in the multivariate regression are collected by roughly approximating the shock location for a known air throttle valve opening and determining which transducers display a disturbed output. The shock is said to be located at the location of the last transducer that exhibits disturbance. A method similar to the shock location determination for $X_{s}$ is not feasible here due to the near instantaneous change in output when the air throttle is opened. The shock location determined here is thus knowingly underestimated and imprecise, but will suffice for the purposes of estimating $X_{\Delta}$. Figure 4.3 shows the data collected and an exponential trend that captures its behavior. The exponential function determined by the data is used as the $f_{3}$ expression in the nonlinear output function to calculate an 
estimate for $X_{\Delta}$. Details of the exponential fit are given in Appendix C.

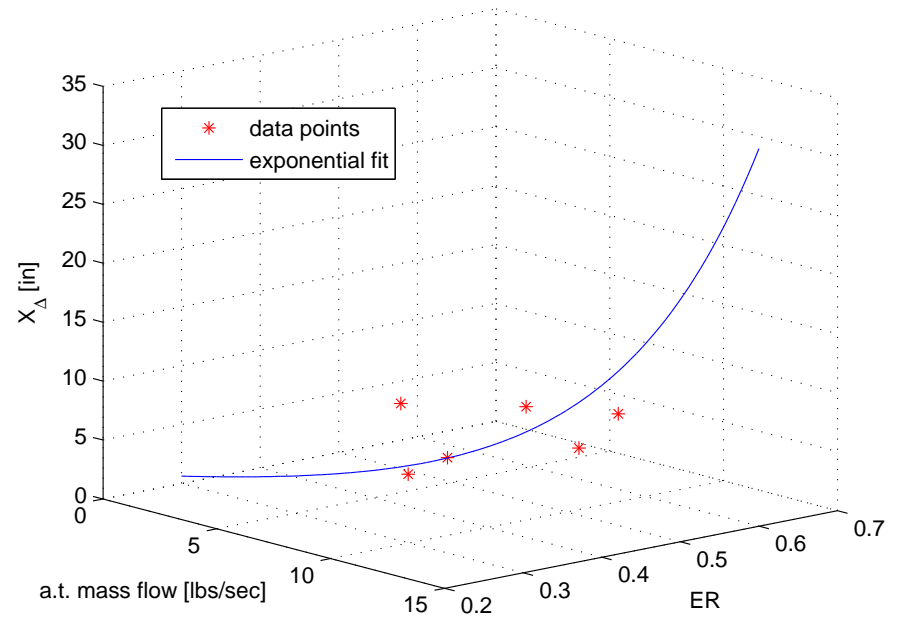

Figure 4.3: Exponential fit describing equivalence ratio and air throttle vs.

$$
X_{\Delta}, R^{2}=0.759
$$

\section{Output scaling when air throttle advances shock past transducer}

When the pressure at a transducer location is disturbed only because of the extra shock motion induced by the air throttle, the output must be appropriately scaled based on the distance between the transducer's location $X_{t}$ and the equivalence ratio shock location $X_{s}$ as well as the magnitude of the air throttle. The data points used to facilitate the multivariate regression are easily collected as $X_{t}$ and the air throttle are exactly known and $X_{s}$ is directly calculated from the equivalence ratio. Figure 4.4 displays a linear trend that accommodates the data collected. The function is used as the $f_{1}$ expression in the nonlinear output function to scale the pressure output in the $X_{\Delta}$-influenced cases. Details of the linear fit are given in Appendix C. 


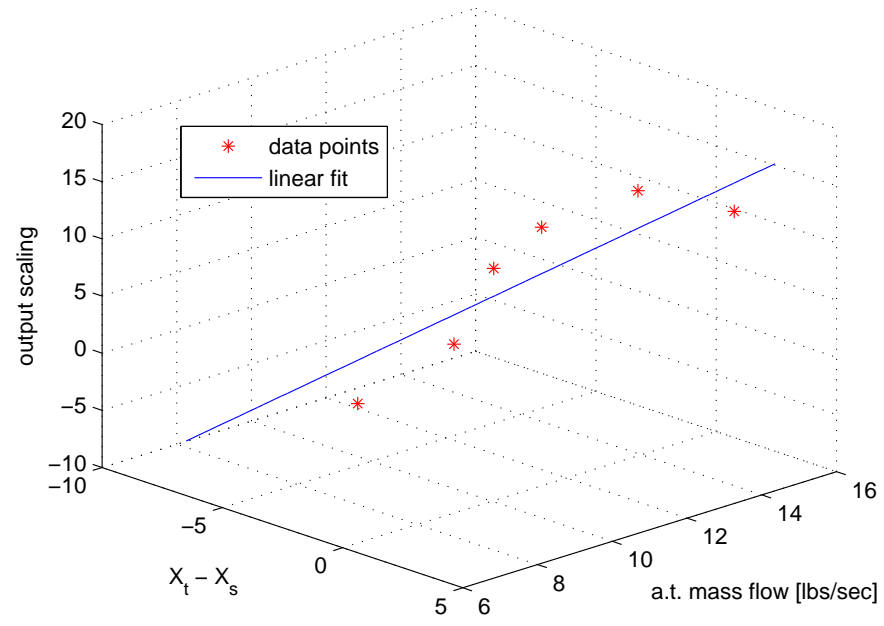

Figure 4.4: Linear fit describing distance from equivalence ratio-based shock to selected transducer and air throttle vs. pressure output scaling,

$$
R^{2}=0.887
$$

\subsubsection{Results}

The output prediction results of the simplified model are shown in the following figures. The same runs used to demonstrate the full system identification model are also used here. Figures 4.5 and 4.6 illustrate the two runs used for the system identification of the linear portion of the model. The transducer selected from each run was chosen based on the fact that its location did not experience any of the identified nonlinear factors. Thus the linear model is generated from approximately linear data and the output is adequately predicted in each case. A first order model was used as higher orders offered no substantial improvements to output matching. 


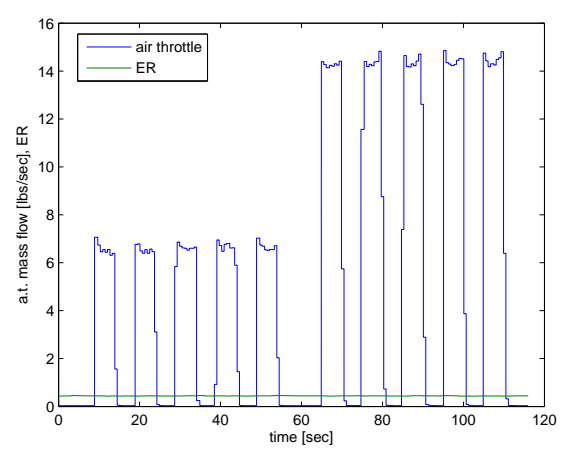

(a) Input

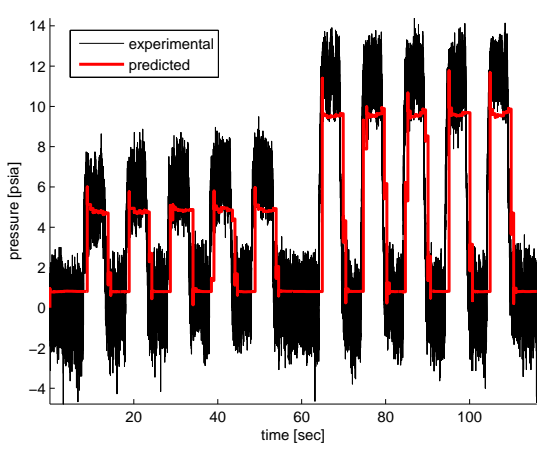

(b) Output

Figure 4.5: First model generation run

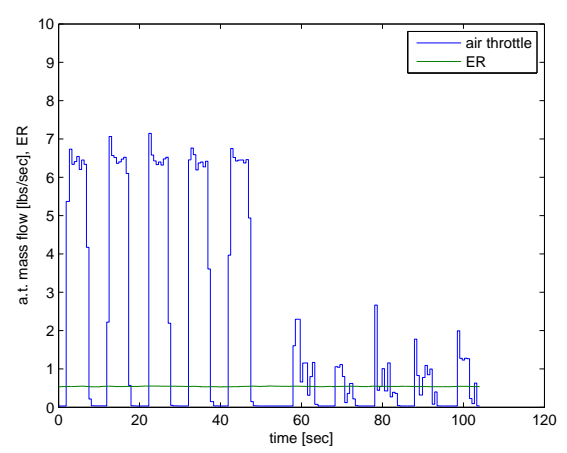

(a) Input

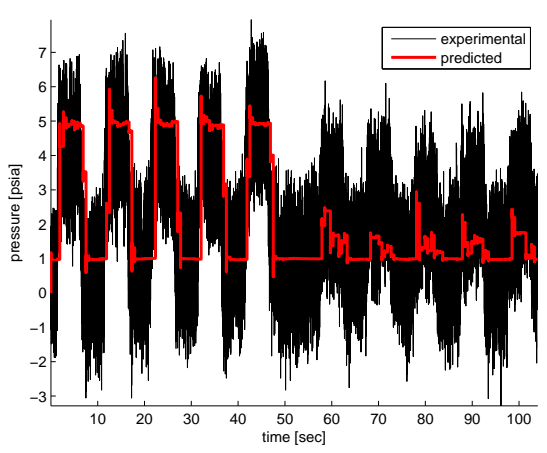

(b) Output

Figure 4.6: Second model generation run 
Figure 4.7 shows the output predicted for a separate run using the entire simplified model (linear block and previously determined nonlinear output function). This validation run was not used as one of the linear block generation runs, and the model is blindly predicting the pressure output based only on the input signals. The transducer selected was chosen based on the fact that all of the nonlinear factors are encountered at its location during the course of the run. The predicted output clearly matches the experimental output very well. Pressure changes are only predicted when appropriate. When the signal is noisy, the magnitude of the prediction approximately matches the mean value of the pressure. The significant delay previously noticed when using the full system identification model is also drastically reduced here with the use of the simplified model. Any prediction delay that is still seen is due directly to the lower sampling of the input signals as closer examination reveals that the predicted output responds to changes in the input (when appropriate) at the same time step they occur.

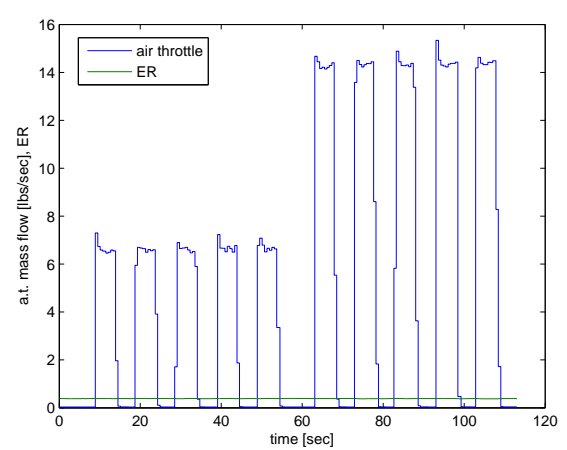

(a) Input

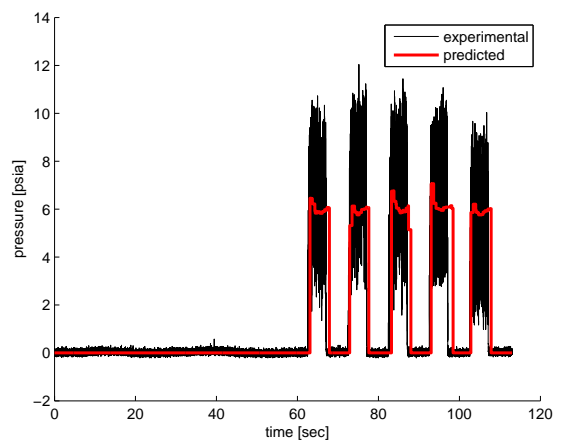

(b) Output

Figure 4.7: Model validation run 
Because the relationships in the simplified model's nonlinear output function are calculated directly from data, the model structure is likely to be successful only on runs from the same data set. This limitation is not seen with the full system identification model structure as even though the model parameters would be reselected during the identification process, the structure for the blocks would not change. Despite this loss of generality, additional functionality is gained with the ability to use the model structure at any transducer location. The simplified model makes use of the transducer's location in the nonlinear output function meaning that the function can be appropriately adjusted when applied at a new location without altering the structure. Figure 4.8 illustrates the simplified model blindly applied to another transducer location from the same run shown in Figure 4.7. Again the predicted output matches the experimental output and displays the same desirable characteristics.

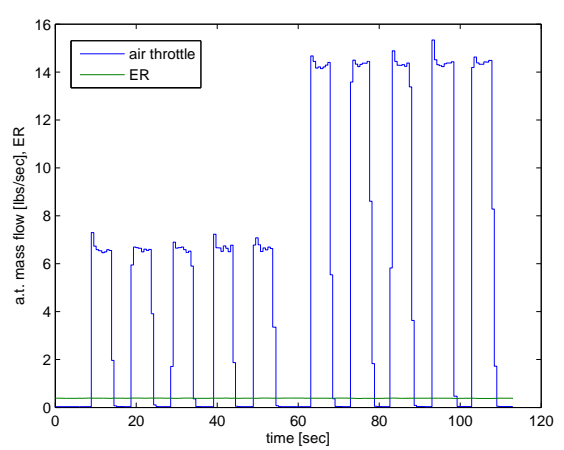

(a) Input

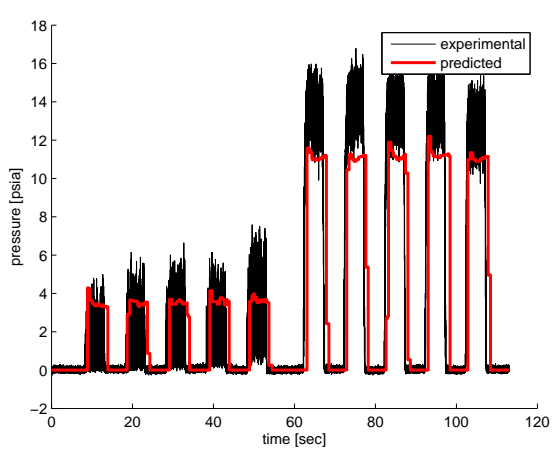

(b) Output

Figure 4.8: Model validation run, second transducer location 


\section{Chapter 5}

\section{Conclusions and Suggestions for Future Work}

In this thesis, the topics of unstart detection and low-dimensional modeling of associated transient dynamics have been addressed. Concerning detection of shock location and ultimately unstart, the CUSUM and other algorithms together offer a reliable means of determining abrupt changes in pressure data. They provide a way to rapidly and autonomously determine when the shock passes a transducer. However, these algorithms do not currently provide any functionality to detect the approaching shock ahead of time. With regard to modeling the transient dynamics of shock motion, preliminary success has been achieved by way of models that can predict the pressure at a sensing location with the specific types of inputs investigated. Models generated entirely by system identification were successful but unnecessary as they revealed that the nonlinear and linear dynamics could be easily separated and modeled individually. A simplified nonlinear model achieved through this separation was shown to be largely successful and was additionally shown to resolve some of the issues facing the full system identification models.

Future efforts in detection should be guided towards finding a consistent precursor of the approaching shock and a means to detect it. Other work 
exists in this area and the results of some such work have been considered here in the form of power spectrum based detection [14], [12]. However, none of the precursors have yet shown to provide a consistent, significant feature to use for detection over a wide range of conditions. The estimated dynamic models also lack generality that should be addressed in the future as their success is limited primarily to runs generated with similar input patterns and disturbance sources. While it is not expected that a single model will be found that can universally accommodate all unstart data, ideally one model should at least be able to accommodate all data generated with similar geometry and flow conditions. To accomplish this, a wider variety of runs with differing input patterns will have to be incorporated into model generation. Also, current models rely on the fact that sources intended to act as disturbances (i.e. air throttle, flap) are modeled as inputs. This dependency with have to be removed or modified to work with more realistically available in-flight information.

Ultimately, the two topics of this thesis should be used concurrently to simulate in-flight operation and facilitate closed-loop control design. As the model permits simulation of the transient dynamics for the shock system, it is possible to generate the output expected for planned mission inputs, run a change detection scheme on the predicted output, and determine when decision to actuate would practically occur. The time determined for actuation can guide requirements on actuator capability and in turn influence development of a feedback control law in preparation for full-scale experimental implementation of closed-loop control. 
Appendices 


\section{Appendix A}

\section{UT Full System Identification Model}

The linear portion of the UT data's Hammerstein-Wiener model is given by

$$
y(t)=\frac{B(q)}{F(q)} u(t)+e(t)
$$

where the numerator and denominator are given for each input/output pair by the following relationships:

$$
\begin{aligned}
& B_{1}(q)=-0.5245 q^{-1}+0.02273 q^{-2}+q^{-3}-0.001136 q^{-4}-0.4975 q^{-5} \\
& B_{2}(q)=0.48785 q^{-1}-0.9152 q^{-2}-0.1053 q^{-3}+q^{-4}-0.4673 q^{-5} \\
& F_{1}(q)=1+0.1281 q^{-1}-1.916 q^{-2}-0.3211 q^{-3}+0.9583 q^{-4}+0.1508 q^{-5} \\
& F_{2}(q)=1-1.174 q^{-1}-1.529 q^{-2}+1.869 q^{-3}+0.5496 q^{-4}-0.7158 q^{-5}
\end{aligned}
$$

The piecewise nonlinearity estimators at the input and output of the model's linear system are defined by breakpoints $\left(x_{k}, y_{k}\right)$ where $k$ ranges from 1 to the number of piecewise units. The breakpoints define an estimator of the

form $y_{k}=F\left(x_{k}\right)$ where $F$ is linearly interpolated in between each set of points. The breakpoints for the input piecewise nonlinearities (one for each input) are given in Tables A.1 and A.2. The breakpoints for the output piecewise nonlinearity is given in Table A.3. 
Table A.1: 10 unit piecewise nonlinearity for input 1

\begin{tabular}{|c|cccccccccc|}
\hline Unit & 1 & 2 & 3 & 4 & 5 & 6 & 7 & 8 & 9 & 10 \\
\hline$x_{k}$ & 1.545 & 3.091 & 4.636 & 6.182 & 7.727 & 9.273 & 10.818 & 12.364 & 13.909 & 15.455 \\
$y_{k}$ & 0.403 & 0.432 & 0.572 & 0.801 & 1.098 & 1.441 & 1.810 & 2.183 & 2.538 & 2.854 \\
\hline
\end{tabular}

Table A.2: 10 unit piecewise nonlinearity for input 2

\begin{tabular}{|c|cccccccccc|}
\hline Unit & 1 & 2 & 3 & 4 & 5 & 6 & 7 & 8 & 9 & 10 \\
\hline$x_{k}$ & 0.097 & 0.187 & 0.277 & 0.366 & 0.456 & 0.546 & 0.636 & 0.725 & 0.815 & 0.905 \\
$y_{k}$ & -0.129 & -0.089 & -0.014 & 0.087 & 0.205 & 0.332 & 0.461 & 0.583 & 0.690 & 0.773 \\
\hline
\end{tabular}

Table A.3: 10 unit piecewise nonlinearity for output

\begin{tabular}{|c|cccccccccc|}
\hline Unit & 1 & 2 & 3 & 4 & 5 & 6 & 7 & 8 & 9 & 10 \\
\hline$x_{k}$ & -1.029 & 0.146 & 1.611 & 3.085 & 4.559 & 6.035 & 7.532 & 8.982 & 10.431 & 11.881 \\
$y_{k}$ & 0.597 & -1.030 & -2.730 & -4.796 & -5.781 & -6.539 & -11.691 & -80.361 & -146.848 & -145.290 \\
\hline
\end{tabular}




\section{Appendix B}

\section{AFRL Full System Identification Model}

The linear portion of the AFRL data's Hammerstein-Wiener model is given by

$$
y(t)=\frac{B(q)}{F(q)} u(t)+e(t)
$$

where the numerator and denominator are given for each input/output pair by the following relationships:

$$
\begin{aligned}
& B_{1}(q)=-0.6196+q^{-1}-0.4516 q^{-2}-0.04826 q^{-3} \\
& B_{2}(q)=-0.626+q^{-1}+0.08246 q^{-2}-0.4378 q^{-3} \\
& F_{1}(q)=1-1.444 q^{-1}+0.4826 q^{-2}+0.1939 q^{-3} \\
& F_{2}(q)=1-0.165 q^{-1}-0.7045 q^{-2}-0.04938 q^{-3}
\end{aligned}
$$

The model's input nonlinearity estimator is described by a sigmoid network of the form

$$
F(x)=(x-r) P L+a_{1} f\left((x-r) Q b_{1}+c_{1}\right)+\ldots+a_{n} f\left((x-r) Q b_{n}+c_{n}\right)+d
$$

where $f$ is the sigmoid function given by

$$
f(z)=\frac{1}{e^{-z}+1} .
$$


The parameter values of the sigmoid network for input 1 are given in Table B.1. The parameter values for input 2 are given in Table B.2.

The output nonlinearity estimator is defined by a deadzone function. This nonlinear function has the form $y=F(x)$ where $\mathrm{F}$ is a function of $x$ and has the following characteristics:

$$
\begin{aligned}
& a \leq x<b \rightarrow F(x)=0 \\
& x<a \rightarrow F(x)=x-a \\
& x \geq b \rightarrow F(x)=x-b .
\end{aligned}
$$

Here $a=-2.3909$ and $b=18.0043$. 
Table B.1: Sigmoid network parameter values for input 1

\begin{tabular}{|c|c|c|}
\hline Name & Variable & Value \\
\hline Regressor Mean & $r$ & 4.7351 \\
\hline Nonlinear Subspace & $Q$ & 1 \\
\hline Linear Subspace & $P$ & 1 \\
\hline Linear Coefficient & $L$ & -3.0880 \\
\hline Dilation & $b_{n}$ & $\begin{array}{c}24.4633 \\
37.3450 \\
42.8350 \\
32.8013 \\
12.3858 \\
5752.4319 \\
130.8139 \\
82.3848 \\
-188.6843 \\
51.7753\end{array}$ \\
\hline Translation & $c_{n}$ & $\begin{array}{c}216.3860 \\
277.0965 \\
297.9520 \\
231.2517 \\
181.3906 \\
-3634.5595 \\
-233.8980 \\
-99.2769 \\
4176.58398 \\
-384.7245\end{array}$ \\
\hline Output Coefficient & $a_{n}$ & $\begin{array}{c}-21781.9423 \\
49855.1023 \\
-36810.2482 \\
8697.5432 \\
9.7585 \\
5.6497 \\
0.7205 \\
-0.8188 \\
-0.2280 \\
4.3581\end{array}$ \\
\hline Output Offset & $d$ & -7.1532 \\
\hline
\end{tabular}


Table B.2: Sigmoid network parameter values for input 2

\begin{tabular}{|c|c|c|}
\hline Name & Variable & Value \\
\hline Regressor Mean & $r$ & 0.4422 \\
\hline Nonlinear Subspace & $Q$ & 1 \\
\hline Linear Subspace & $P$ & 1 \\
\hline Linear Coefficient & $L$ & 472.3702 \\
\hline Dilation & $b_{n}$ & $\begin{array}{c}1464.2527 \\
-732421.1369 \\
152.6816 \\
607.4544 \\
20995.7966 \\
7928.0564 \\
57691.1465 \\
9855.9349 \\
868.9129 \\
-15720.6167\end{array}$ \\
\hline Translation & $c_{n}$ & $\begin{array}{c}8.0954 \\
-5558.9950 \\
11.2937 \\
96.0073 \\
-1.8098 \\
127.5857 \\
-116.2601 \\
6.1043 \\
-8.3168 \\
152.7896\end{array}$ \\
\hline Output Coefficient & $a_{n}$ & $\begin{array}{c}16.3813 \\
19.6733 \\
5.6568 \\
-27.2492 \\
-10.6395 \\
16.0689 \\
-5.9660 \\
14.1569 \\
-5.8649 \\
-3.3694\end{array}$ \\
\hline Output Offset & $d$ & -7.6356 \\
\hline
\end{tabular}




\section{Appendix C}

\section{AFRL Simplified Model}

The linear portion of the simplified model is described by Equation (C.1). In this model there are two inputs: the air throttle mass flow rate and the equivalence ratio. The output is the pressure at the selected transducer.

$$
\begin{gathered}
x\left(t+T_{s}\right)=A x(t)+B u(t) \\
y(t)=C x(t)+D u(t)
\end{gathered}
$$

System identification produced a first-order model with the necessary matrices specified in Equation (C.2).

$$
\begin{gathered}
A=[-0.20153] \\
B=[0.00048758 \quad 0.001408] \\
C=[1513.9] \\
D=\left[\begin{array}{ll}
0 & 0
\end{array}\right]
\end{gathered}
$$

The output nonlinearity is determined based on two factors: shock location due to equivalence ratio, $\phi$, and shock motion due to air throttle disturbance, $a$. For the equivalence ratio consideration the shock location 
due only to the equivalence ratio portion of the input, $X_{s}$, is determined by Equation (C.3) which is calculated at each of the $k$ sample times.

$$
X_{s, k}=10.259+95.138 \phi_{k}-147.78 \phi_{k}^{2}
$$

If the equivalence ratio shock location is behind the selected transducer (i.e. $X_{s}>X_{t}$ ) then calculation of the output will require consideration of the air throttle disturbance. Otherwise, the output is calculated according to Equation (C.1).

In the case that the shock location is behind the selected transducer only then is the air throttle nonlinearity considered. If this is the case then the change in shock location $X_{\Delta}$ from $X_{s}$ is calculated according to Equation (C.4). Note that this equation depends on both inputs as the impact of the air throttle changes throughout the inlet. The dependence on equivalence ratio is an indirect way of stating the approximate location within the inlet for this data set.

$$
X_{\Delta, k}=0.094406\left(1.14658^{a_{k}}\right)\left(539.9836468^{\phi_{k}}\right)
$$

If the shock location now moves beyond the selected transducer (i.e. $\left(X_{s}-\right.$ $\left.X_{\Delta}\right)<X_{t}$ ) then the output is calculated according to Equation (C.5). Otherwise the output is set to 0 . This equation acts as a scaling factor for output that is only effected by the the air throttle. As the impact of the air throttle varies, the scaling depends on the air throttle disturbance itself as well as distance between $X_{s}$ and $X_{t}$. Inclusion of $X_{s}$ is again an indirect way of stating 
the approximate location within the inlet involved.

$$
y_{k}=-2.87368+1.283582\left(X_{t}-X_{s, k}\right)+0.999005 a_{k}
$$




\section{Bibliography}

[1] D. Andreadis. Scramjets integrate air and space. Industrial Physicist, pages 26-29, 2004.

[2] William H. Heiser and David T. Pratt. Hypersonic Airbreathing Propulsion. AIAA, 1994.

[3] E. T. Curran and F. E. Stull. The utilization of supersonic combustion ramjet systems at low mach numbers. Technical report, Aero Propulsion Lab, RTD-TDR-63-4097, Wright-Patterson AFB, OH, January 1964.

[4] E. T. Curran, W. H. Heiser, and D. T. Pratt. Fluid phenomena in scramjet combustion systems. Annual review of Fluid Mechanics, 28(1):323360, 1996.

[5] D. M. Van Wie, F. T. Kwok, and R. F. Walsh. Starting characteristics of supersonic inlets. In AIAA, ASME, SAE, and ASEE Joint Propulsion Conference and Exhibit, July 1996.

[6] Miklos Sajben, John F. Donovan, and Martin J. Morris. Experimental investigation of terminal shock sensors for mixed-compression inlets. Journal of Propulsion and Power, 8(1):168-174, 1992. 
[7] D. B. Lee, C. P. Goyne, R. H. Krauss, and J. C. McDaniel. Experimental study of a dual-mode scramjet isolator. Journal of Propulsion and Power, 24(5):1050-1057, 2008.

[8] Jeffrey M. Donbar, Graham J. Linn, Sukumar Srikant, and Maruthi R. Akella. High-frequency pressure measuremnts for unstart detection in scramjet isolators. In 46th $A I A A / A S M E / S A E / A S E E$ Joint Propulsion Conference $\&$ Exhibit, July 2010.

[9] G. B. Rieker, J. B. Jeffries, R. K. Hanson, T. Mathur, M. R. Gruber, and C. D. Carter. Diode laser-based detection of combustor instabilities with application to a scramjet engine. Proceedings of the Combustion Institute, 32(1):831-838, 2009.

[10] Mark Gruber, Campbell Carter, Michael Ryan, Gregory B. Rieker, Jay B. Jeffries, Ronald K. Hanson, Jiwen Liu, and Tarun Mathur. Laserbased measurements of $\mathrm{OH}$, temperature, and water vapor concentration in a hydrocarbon-fueled scramjet. In $A I A A / A S M E / S A E / A S E E$ Joint Propulsion Conference $\mathfrak{E}$ Exhibit, July 2008.

[11] D. B. Le, C. P. Goyne, and R. H. Krauss. Shock train leading-edge detection in a dual-mode scramjet. Journal of Propulsion and Power, 24(5):1035-1041, 2008.

[12] S. Srikant, J. L. Wagner, A. Valdivia, M. R. Akella, and N. Clemens. Unstart detection in a simplified-geometry hypersonic inlet-isolator flow. Journal of Propulsion and Power, 26:1059-1071, 2010. 
[13] John R. Hutzel, Douglas D. Decker, Richard G. Cobb, Paul I. King, and Michael J. Veth. Scramjet isolator shock train location techniques. In 49th AIAA Aerospace Sciences Meeting, January 2011.

[14] S. Trapier, S. Deck, P. Duveau, and P. Sagaut. Time-frequency analysis and detection of supersonic inlet buzz. AIAA Journal, 45(9):2273-2283, 2007.

[15] Hui-jun Tan, Liu-gang li, Yu-fen Wen, and Qi-fan Zhang. Experimental investigation of the unstart process of a generic hypersonic inlet. AIAA Journal, 49(2):279-288, February 2011.

[16] A. Valdivia, K.B. Yuceil, J. L. Wagner, N. T. Clemens, and D. S. Dolling. Active control of supersonic inlet unstart unsing vortex generator jets. In 39th AIAA Fluid Dynamics Conference, June 2009.

[17] Seong-kyun Im, Hyungrok Do, and Mark A. Cappelli. Plasma control of an unstarting supersonic flow. In 17th AIAA International Space Planes and Hypersonic Systems Technologies Conference, April 2011.

[18] Pratik Donde, Heeseok Koo, and Venkatramanan Raman. Large eddy simulation of supersonic combustion using direct quadrature method of moments. In AIAA Aerospace Sciences Meeting Including the New Horizons Forum and Aerospace Expostions, January 2010.

[19] Cui Tao, Yu Daren, Chang Junator, and Bao Wen. Topological geometry interpretation of supersonic inlet start/unstart based on catastrophe 
theory. Journal of Aircraft, 45(4):1464-1468, August 2008.

[20] John R. Hutzel, Douglas D. Decker, and Jeffrey M. Donbar. Scramjet isolator shock-train leading-edge location modeling. In 17th AIAA International Space Planes and Hypersonic System and Technologies Conference, April 2011.

[21] S. Smith, M. Gruber, R. Steiner, M. Collatz, and T. Mathur. Development and calibration of an axisymmetric direct-connect supersonic combustion flowpath. In 45th AIAA/ASME/SAE/ASEE Joint Propulsion Conference ES Exhibit, August 2009.

[22] E. S. Page. Continuous inspection schemes. Biometrika, 41:100-114, June 1954.

[23] Nl L. Johnson. A simple theoretical approach to cumulative sum control charts. Journal of the American Statistical Association, 56:835-840, 1961.

[24] Juho Vihonen, Juha Jylha, Timo Ala-Kleemola, Marga Ruotsalainen, Jarmo Kauppila, Tommi Huotilainen, Juhani Rauhamaa, and Ari Visa. Directional filtering for sequential image analysis. IEEE Signal Processing Letters, 15:902-905, 2008.

[25] J. Dukich and D. Hawkins. Identifying shifts in spread using the cauchy cusum: An application to the japanese yen/us dollar exchange rate. $A p$ plied Financial Economics, 20(5):417-424, 2010. 
[26] Stefan H. Steiner and Richard J. Cook. Monitoring surgical performance using risk-adjusted cumulative sum charts. Biostatistics, 1(4):441-452, 2000 .

[27] L. Ljung. System Identification: Theory for the User. Prentice Hall, second edition, 1999.

[28] Compare model output and measured output-MATLAB. http://www. mathworks.com/help/toolbox/ident/ref/compare.html, 2011.

[29] E.W. Bai. A blind approach to the Hammerstein-Wiener model identification. Automatica, 38(6):967-979, 2002.

[30] Hammerstein-Wiener model-MATALB. http://www.mathworks.com/help /toolbox/ident/gs/bqzx4jq-5.html\#bqz8hri, October 2011.

[31] Oliver Nelles. Nonlinear System Identification. Springer, 2001.

[32] Karel J. Keesman. System Identification: An Introduction. Springer, 2011. 